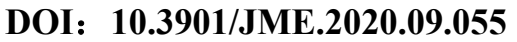

\title{
非圆齿轮设计、制造、检测及应用*
}

\author{
李渤涛 陈定方 \\ (武汉理工大学智能制造与控制研究所 武汉 430063)
}

\begin{abstract}
摘要: 非圆齿轮最早可追溯到 14 世纪左右, 如今已有 700 多年历史。然而非圆齿轮的研究历史却并不那么久远, 受限于非 圆齿轮特殊的几何特性及落后的工业技术水平, 非圆齿轮在过去一直未被广泛应用, 相关研究亦少之又少。进入 21 世纪后, 计算机辅助设计制造技术的迅猛发展, 给非圆齿轮领域带来了新的活力, 涌现出大量新的研究成果。鉴于此, 归纳总结现阶 段非圆齿轮(以非圆柱状齿轮为主)研究现状、预测非圆齿轮领域未来发展方向是很有意义的。现阶段, 非圆齿轮领域主要可 分为设计与分析、制造、检测及应用四个主要研究领域, 通过对上述领域常用理论与方法的总结, 可以看出各领域现阶段所 面对的问题，预测今后发展方向，以期启发非圆齿轮领域的研究者们，进一步推动非圆齿轮发展与应用。
\end{abstract}

关键词: 非圆齿轮; 设计与分析; 制造; 检测; 应用

中图分类号: TH11

\section{Design, Manufacture, Inspection and Application of Non-circular Gears}

\section{Botao CHEN Dingfang}

(Institute of Intelligent Manufacturing and Control, Wuhan University of Technology, Wuhan 430063)

\begin{abstract}
Non-circular gears appeared as early as the 14th century and have a history of more than 700 years. However, the research of non-circular gears is not as long as its history. Due to the special geometry of non-circular gears and the restriction of the design, analysis, manufacture and testing technology in the past, non-circular gears have not been widely used. Nowadays, with the development of computer technology, non-circular gears have again attracted the researchers' attention. In many studies, non-circular gears have applied to more and more application areas. In order to inspire researchers in this field and expand the application field of non-circular gears, the background and development of non-circular gears (mainly cylindrical gears) in the four main research fields: design and analysis, manufacture, testing and application have been summarized and analyzed. Then, the advantages and disadvantages of various theories and methods in the above research fields have been summarized, as well as the problems faced and the possible future development directions.
\end{abstract}

Key words: non-circular gear; design and analysis; manufacturing; testing; application

\section{0 前言}

非圆齿轮，因其具有结构紧凑、动平衡好、传 动可靠、效率高、承载能力强、精度高等优点, 广 泛应用于农业、纺织、仪器仪表等领域 ${ }^{[1]}$ 。早在 14 世纪，天文钟中就采用了高精度非圆齿轮机构 ${ }^{[2]}$ 。 进入 20 世纪后, 为了实现复杂的变速比传动, 满足 机械制造业对传动机构多样化、复杂化的需要, DUNKELEY 在其著作《Mechanism》中系统提出了

\footnotetext{
* 国家自然科学基金(51805395)和 2019 年湖北省技术创新专项(重大专 项)(ZDCX2019000018)资助项目。20190612 收到初稿, 20191025 收到 修改稿
}

椭圆齿轮的概念。此后, 各国学者相继开展了非圆 齿轮的研究工作, 其中, 苏联学者 LITVIN 在《齿 轮啮合原理》中, 给出了非圆齿轮详细设计原理与 加工方法; 日本学者香取英男等开发了自动设计、 自动生成数控加工指令、自动检验为一体的非圆齿 轮设计、加工、检测程序包; 德国、法国、加拿大 等国的学者也相继在非圆齿轮的设计与分析、制造、 检测与应用方面进行了深入研究, 并开发了相应的 计算机辅助设计/制造程序包。

20 世纪 80 年代以来, 非圆齿轮在我国也掀起 了一波研究热潮。李福生等在 1973 年翻译了 LITVIN 所著的《非圆齿轮》, 又在 1981 年编著了《非 圆齿轮与特种齿轮传动设计》; 吴序堂等在 1996 年 编著了《非圆齿轮及非匀速比传动》。这几本著作详 
细地介绍了非圆齿轮设计、制造及检测。同一时期, 国内其他研究者亦在非圆齿轮的设计、制造及应用 领域做出了诸多贡献。这些成果缩小了我国非圆齿 轮传动技术研究与应用与世界的差距, 为我国非圆 齿轮的后续研究打下了坚实基础。

但在当时, 受设计与制造水平制约, 非圆齿轮 设计、制造难度大, 成品精度低, 难以满足实际应 用需要, 限制了非圆齿轮的广泛应用, 相关研究也 越来越少。直到步入 21 世纪, 随着计算机技术的不 断发展、 $\mathrm{CAD} / \mathrm{CAM}$ 技术的广泛应用 ${ }^{[3]}$, 打破了非 圆齿轮设计难、制造难的瓶颈, 使其再次回到了研 究者们的视野中。目前, 非圆齿轮有关的研究仍以 非圆柱状齿轮为主, 主要集中在设计与分析、制造、 检测及应用这 4 个研究领域。下面将以非圆齿轮这 4 个领域为主, 综合分析国内外相关研究, 简要概 括各领域现阶段主要研究理论、方法及结论, 并在 总结与展望中指出各领域中现存的问题及未来可能 的发展方向, 供广大非圆齿轮研究者参考。

\section{1 非圆齿轮设计与分析}

非圆齿轮在传动时具有可变传动比, 部分更有 可变中心距, 为使齿轮对在传动时正确啮合, 非圆 齿轮节曲线与齿廓曲线的设计需要比圆齿轮更加复 杂的设计理论与方法作为支撑。现阶段非圆齿轮节 曲线设计方法主要可分为两类: 灵活运用已知曲线 方程拟合拼接出节曲线的变性拼接拟合法; 以及通过 建立等式关系推算节曲线的微分几何推算法。非圆齿 轮齿廓曲线设计方法现阶段也可大致分为两类: 运用 渐开线齿廓生成原理的渐开线展开法; 以及运用非圆 齿轮范成加工原理反求齿廓曲线的范成法。

\section{1 非圆齿轮节曲线设计}

\subsection{1 曲线变性拼接拟合法}

该方法使用已知解析式的曲线作为基曲线, 通 过调整曲线整解析式中变性系数来改变曲线形状, 必要时还辅以分段、拼接等手段, 将所需非圆齿轮 节曲线拟合出来。对于设计并不复杂的非圆齿轮节 曲线, 是一种简单、高效的方法。其中常用基曲线 有以下几种。

(1) 偏心圆。

现阶段常用的基于偏心圆的非圆齿轮节曲线拟 合公式如下所示 ${ }^{[4]}$

$$
P(\theta)=b \sin ^{k} \theta+e \cos ^{l} \theta+R
$$

该公式是由偏心圆的切线极坐标方程与幂指数 $k 、 l$ 构成。在设计非圆齿轮时, 该公式通过调整变
性系数 $k$ 与 $l$ 生成满足需求的非圆齿轮节曲线方程, 如图 1 所示。

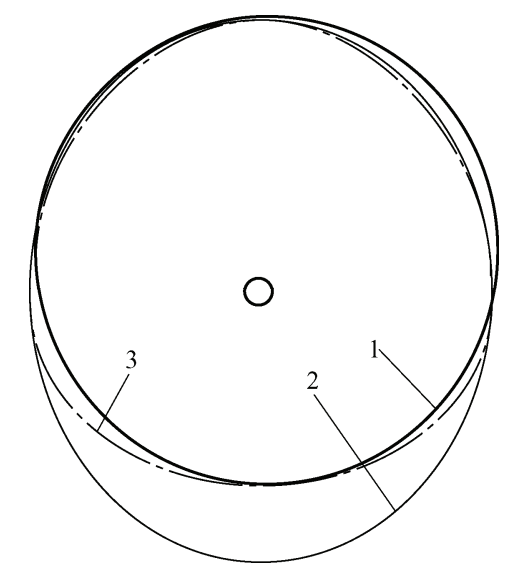

图 $1 k=1 、 2 、 3$ 时的偏心圆节曲线

(2) 高阶椭圆。

现阶段研究中提出了数种基于高阶椭圆的非圆 齿轮节曲线拟合公式 ${ }^{[5-6]}$, 其中, 具有较强通用性的 拟合公式如下所示 ${ }^{[6]}$

$$
\left\{\begin{aligned}
r_{1}= & p_{1} /\left(1-k_{1} \cos n_{1} m_{1} \varphi_{1}\right) \\
& 0 \leqslant \varphi_{1} \leqslant \pi / n_{1} m_{1} \\
r_{2}= & p_{1} /\left(1-k_{1} \cos \left(n_{1} m_{2}\left(2 \pi / n_{1}-\varphi_{1}\right)\right)\right) \\
& \pi / n_{1} m_{1} \leqslant \varphi_{1} \leqslant 2 \pi / n_{1}
\end{aligned}\right.
$$

该公式将高阶变性椭圆曲线在 $\phi=\pi$ 处分为两部 分, 区间 $\phi \in(0 \sim \pi)$ 中的变性系数为 $m_{1}$, 区间 $\phi \in(\pi \sim 2 \pi)$ 中的变性系数为 $m_{2}$ 。通过调整变性系数 $m_{1}$ 与 $m_{2}$, 不仅能生成一般的高阶椭圆节曲线 $\left(m_{1}=m_{2}\right.$ 时), 还能拟合出较为复杂的非圆齿轮节曲线 ${ }^{[7]}\left(m_{1} \neq m_{2}\right.$ 时), 如图 2 所示。

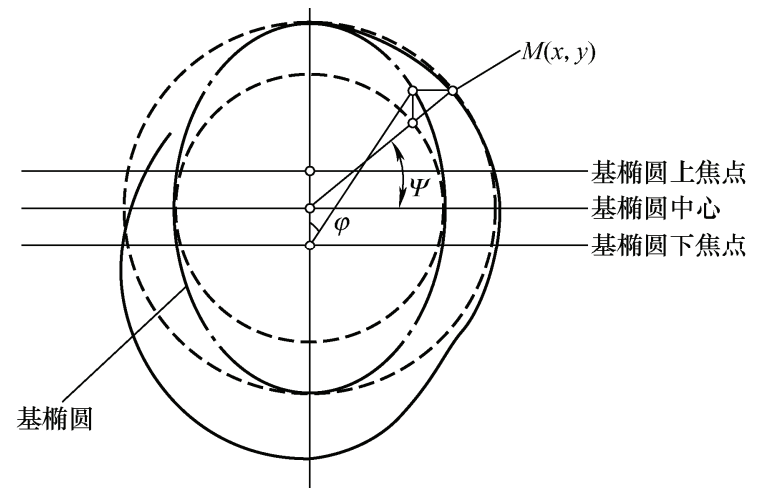

图 $2 n_{1}=2, m_{1}=0.625$ 时的高阶变性椭圆节曲线

除以上两种常见的基曲线形式外, Pascal 蜗线 ${ }^{[8]}$ 、 Hermite 三次参数曲线 ${ }^{[9]}$ 、摆线 ${ }^{[10]}$ 、Aronhold 第一定 理回归圆 ${ }^{[11]}$ 等亦可作为基曲线, 拟合所需的非圆齿 轮节曲线。

为了满足更加复杂的中心距与传动比变化需 求, 部分研究中提出了具有更高灵活性的曲线拟合 
方法, 该方法将非圆齿轮节曲线分为几个部分, 每 部分都寻找与之匹配的基曲线进行拟合, 最后将它们 拼接在一起, 构成满足需求的非圆齿轮节曲线 ${ }^{[11-12]}$ 。 然而, 不同基曲线在拼接点处易出现不连续点及尖 点问题。针对该问题, 又有研究提出了基于样条插 值函数的平滑过渡曲线绘制方法来拼接各段节曲 线、修复不连续点及尖点, 取得了良好成效 ${ }^{[13]}$ 。

\subsection{2 微分几何推算法}

微分几何推算法在已知传动比与中心距变化需 求的情况下能准确设计出满足需求的非圆齿轮节曲 线, 是非圆齿轮节曲线设计的一般化方法。该方法 以啮合原理为基础, 依据非圆齿轮传动比、中心距 变化曲线建立几何关系式, 通过一系列公式推导计 算出主/从动轮各转角所对应的啮合点坐标, 如图 3 所示。再通过坐标变换将啮合点坐标变换为主/从动 轮节曲线上的离散点, 最后经样条插值, 即可反求 出非圆齿轮主/从动轮节曲线。然而, 传统微分几何 推算法理论难度较高, 啮合点坐标难以计算, 尤其 是在非圆齿轮啮合时中心距可变的情况下, 啮合点 坐标需要求解高阶偏微分方程才能得到, 计算过程 复杂, 且存在无法解出的可能 ${ }^{[14]}$ 。为了解决传统微 分几何法难以求得非圆齿轮节曲线的问题, 部分研究 给出了运用旋量工具求解非圆齿轮节曲线的方法, 相 比高阶偏微分公式, 旋量公式更易解出节曲线方程, 但由于旋量理论并不如传统方法直观, 因此现阶段尚 未被广泛关注, 现阶段相关研究还比较少。

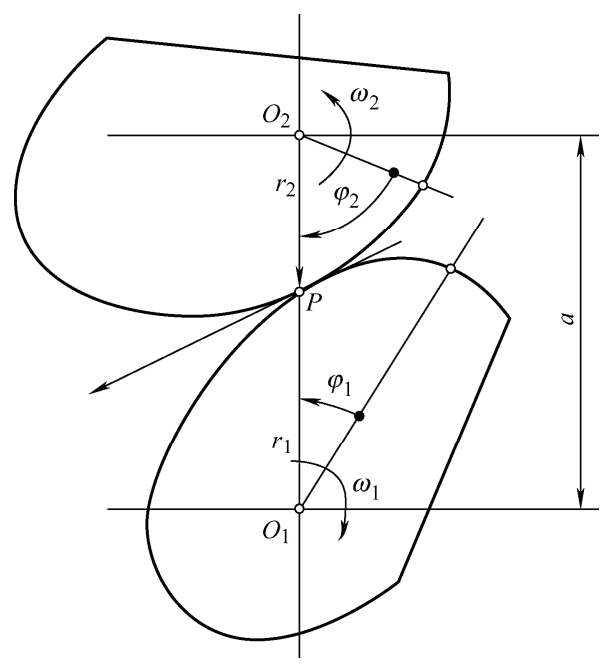

图 3 非圆齿轮啮合图

(1) 传统微分几何推算法。

使用传统微分几何推算法计算定中心距非 圆齿轮节曲线的主要步骤可概括如下。在已知定 中心距非圆齿轮传动比情况下, 其传动比函数可 表示为

$$
i_{12}\left(\varphi_{1}\right)=\omega_{1}\left(\varphi_{1}\right) / \omega_{2}\left(\varphi_{2}\right)
$$

则依据齿轮啮合原理, 当非圆主动轮转角为 $\varphi_{1}$ 时, 可计算出主动轮与从动轮在啮合点处的转动半径 $r_{1}\left(\varphi_{1}\right)$ 与 $r_{2}\left(\varphi_{1}\right)$ 如式(4)、(5)所示

$$
\begin{gathered}
r_{1}\left(\varphi_{1}\right)=a /\left(1+i_{12}\left(\varphi_{1}\right)\right) \\
r_{2}\left(\varphi_{1}\right)=a-a /\left(1+i_{12}\left(\varphi_{1}\right)\right)
\end{gathered}
$$

可通过啮合点处的转动半径 $r_{1}\left(\varphi_{1}\right)$ 与 $r_{2}\left(\varphi_{1}\right)$ 求 出啮合点坐标, 经过坐标变换反求出主/从动轮节 曲线上的点集, 通过样条拟合得到主/从动轮节 曲线 ${ }^{[15]}$ 。

（2）旋量几何推算法。

除运用传统微分几何理论构建等式外, 部分较 新研究中还提出了一种基于旋量理论建立等式计算 非圆齿轮节曲线的方法, 并将其应用于如图 4 所示 的变中心距非圆齿轮机构节曲线求解上, 该方法不 需要求解高阶偏微分方程, 更易于计算出非圆齿轮 节曲线, 是一种计算非圆齿轮节曲线的新方法 ${ }^{[14]}$ 。 由于定中心距非圆齿轮是变中心距非圆齿轮的一个 特例, 因此下文将以变中心距非圆齿轮为主, 简要 描述旋量理论在非圆齿轮节曲线计算中的应用。

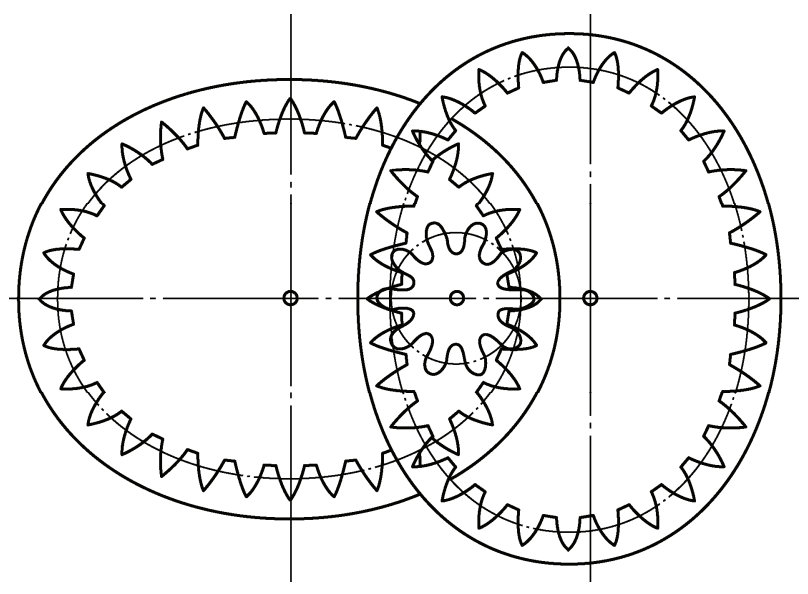

图 4 变中心距非圆齿轮机构

如图 5 所示, 首先在主动轮与从动轮上建立坐标 系 $S_{1}\left(O_{1} x_{1} y_{1} z_{1}\right)$ 与 $S_{2}\left(O_{2} x_{2} y_{2} z_{2}\right)$, 则它们与全局坐标 $S_{0}\left(O_{0} x_{0} y_{0} z_{0}\right)$ 的坐标变换矩阵可表示为 $\boldsymbol{M}_{10}\left(\varphi_{1}\right)$ 与 $\boldsymbol{M}_{20}\left(\varphi_{1}\right)$ 。则主动轮与从动轮轴上的旋量可表示为 ${ }^{[16]}$

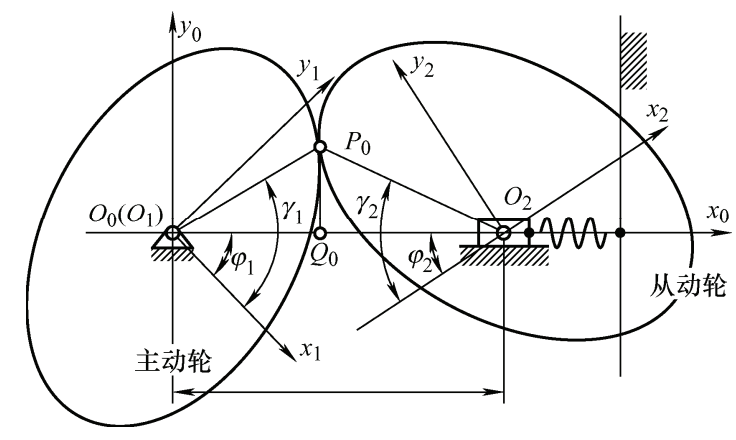

图 5 变中心距非圆齿轮啮合示意图 


$$
\begin{gathered}
\boldsymbol{S}_{\boldsymbol{i}}=\left[\boldsymbol{s}_{i} ; \boldsymbol{s}_{i}\right]=[0,0,1 ; 0,0,0]^{\mathrm{T}} \\
\boldsymbol{S}_{o}=\left[0,0, g\left(\varphi_{1}\right) ; v\left(\varphi_{1}\right),-E\left(\varphi_{1}\right) g\left(\varphi_{1}\right), 0\right]^{\mathrm{T}}
\end{gathered}
$$

主动轮与从动轮在啮合点处的瞬时旋量可表示为

$$
\begin{aligned}
\boldsymbol{S}_{i s}=\boldsymbol{S}_{o}\left(\boldsymbol{\varphi}_{1}\right)-\boldsymbol{S}_{i}= & \\
\quad & {\left[0,0, g\left(\varphi_{1}\right) ; v\left(\varphi_{1}\right),-E\left(\varphi_{1}\right) g\left(\varphi_{1}\right), 0\right]^{\mathrm{T}} } \\
\boldsymbol{S}_{o}= & {\left[0,0, g\left(\varphi_{1}\right), v\left(\varphi_{1}\right),-E\left(\varphi_{1}\right) g\left(\varphi_{1}\right), 0\right]^{\mathrm{T}} }
\end{aligned}
$$

当主动轮转动 $\varphi_{1}$ 时, 主动轮与从动轮上的啮合点坐 标可由极坐标公式表示为

$$
\begin{gathered}
\boldsymbol{O}_{1} \boldsymbol{P}_{0}=\boldsymbol{r}_{u i s}\left(\varphi_{1}\right)=\frac{\boldsymbol{s}_{i s}\left(\varphi_{1}\right) \times \boldsymbol{\zeta}_{i s}\left(\varphi_{1}\right)}{\boldsymbol{s}_{i s}\left(\varphi_{1}\right) \cdot \boldsymbol{s}_{i s}\left(\varphi_{1}\right)}= \\
{\left[\frac{E\left(\varphi_{1}\right) g\left(\varphi_{1}\right)}{g\left(\varphi_{1}\right)-1}, \frac{v\left(\varphi_{1}\right)}{g\left(\varphi_{1}\right)-1}, 0\right]^{\mathrm{T}}} \\
\boldsymbol{O}_{2} \boldsymbol{P}_{0}=\left[E\left(\varphi_{1}\right), 0,0\right]^{\mathrm{T}}-\boldsymbol{r}_{\text {uis }}\left(\varphi_{1}\right)
\end{gathered}
$$

经样条拟合, 即得到主/从动轮节曲线。

\section{2 非圆齿轮齿廓曲线设计}

\subsection{1 渐开线展开法}

LITVIN 在其著作中建议使用渐开线展开法计 算非圆齿轮齿廓曲线 ${ }^{[17]}$, 吴序堂所著《齿轮啮合原 理》中也有描述 ${ }^{[18]}$ 。该方法通过在齿轮基曲线上展 开渐开线的方法获得非圆齿轮齿廓 ${ }^{[19]}$, 符合渐开线 齿廓生成原理, 是非圆齿轮渐开线型齿廓一种合理 的计算方法。但该方法要求首先建立齿轮基曲线。 而有研究认为非圆齿轮基曲线是多义的, 同一节曲

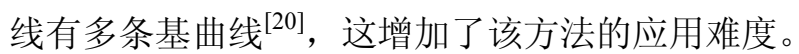
现阶段研究多用节曲线作为非圆齿轮齿廓基曲线, 其计算非圆齿轮齿廓的方法如下 ${ }^{[21]}$

在图 6、7 中, $O$ 为非圆齿轮节曲线圆心; $\theta$ 与 $\phi$ 分别为向径 $\boldsymbol{O A} 、 \boldsymbol{O B}$ 与 $X$ 轴正向的夹角; $\alpha$ 与 $\beta$ 分别为矢量 $\boldsymbol{A E} 、 \boldsymbol{B F}$ 与 $X$ 轴正/负向的夹角; $\tau$ 为节 曲线上过 $A 、 B$ 点的切线与矢量 $\boldsymbol{A} \boldsymbol{E} 、 \boldsymbol{B F}$ 间的夹角, 且其值为 $20^{\circ} ; E 、 F$ 为非圆齿轮节曲线与齿顶/根 曲线间, 左右齿形上任意两点; $C 、 D$ 为节曲线分 别与左右齿形的交点。从图 6、图 7 中可以看出

$$
\begin{aligned}
& \overline{\boldsymbol{A E}}=\overparen{\boldsymbol{A C}} \times \cos \left(20^{\circ}\right) \\
& \overline{\boldsymbol{B F}}=\overparen{\boldsymbol{B D}} \times \cos \left(20^{\circ}\right)
\end{aligned}
$$

则非圆齿轮任意轮齿的左齿廓方程为

$$
O F=O B+B F
$$

即

$$
\left\{\begin{array}{l}
x_{L}=\overline{O B} \times \cos \phi \mp \overline{B F} \times \cos \beta \\
y_{L}=\overline{O B} \times \sin \phi \mp \overline{B F} \times \sin \beta
\end{array}\right.
$$

其右齿廓方程可描述为

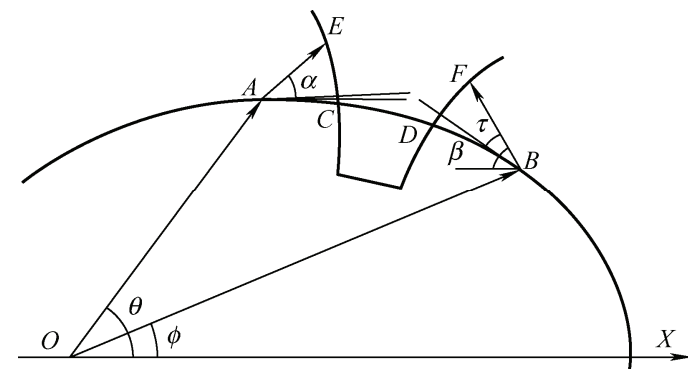

图 6 节曲线与齿顶曲线 1

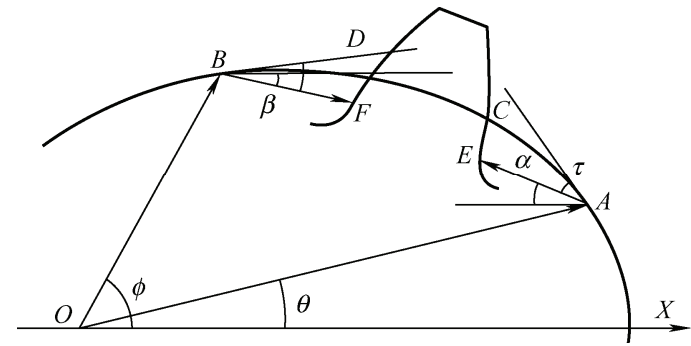

图 7 节曲线与齿根曲线 2

$$
O E=O A+A E
$$

即

$$
\left\{\begin{array}{l}
x_{R}=\overline{O A} \times \cos \theta \mp \overline{A E} \times \cos \alpha \\
y_{R}=\overline{O A} \times \sin \theta \mp \overline{A E} \times \sin \alpha
\end{array}\right.
$$

以上即为渐开线展开法计算出的非圆齿轮齿廓 曲线。

\subsection{2 范成法}

范成法是通过模拟加工过程中产型轮与非圆齿 轮毛胚间的范成运动, 进而求得非圆齿轮齿廓曲线 的一种方法。该方法简单直观，对绝大多数非圆齿 轮都适用, 是现阶段求解非圆齿轮齿廓曲线的主要 方法。该方法按范成方式主要可分为如下两类。

(1) 包络过程中, 齿轮毛胚模型中心固定不动, 毛胚模型绕中心旋转; 刀具模型 (一般为齿条模型) 如图 8 所示配合毛胚模型上下或左右移动, 完成包 络过程 ${ }^{[22]}$ 。

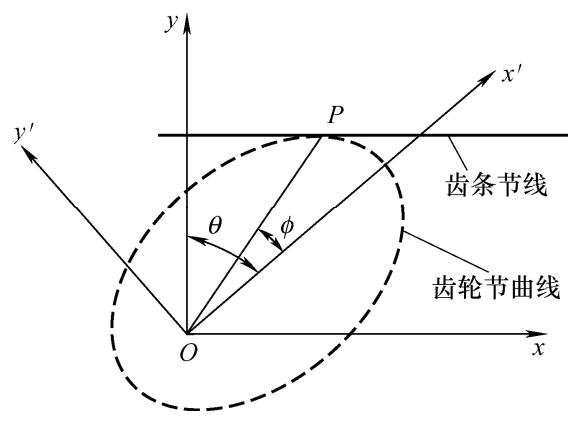

图 8 范成法原理 1

(2) 包络过程中, 非圆齿轮毛胚模型固定不动, 刀具模型(可选用齿条、插齿刀、滚齿刀)如图 9、10 所示通过旋转、平移的方式绕毛胚运动, 完成包络 过程 $[$, 14-15, 23-24]。 


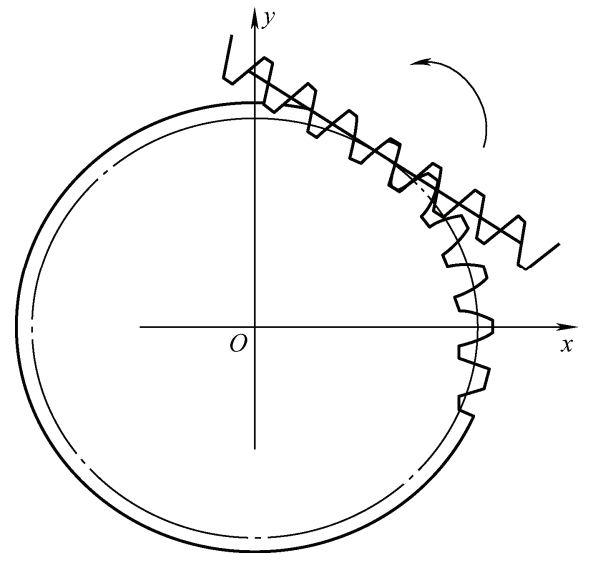

图 9 范成法原理 2

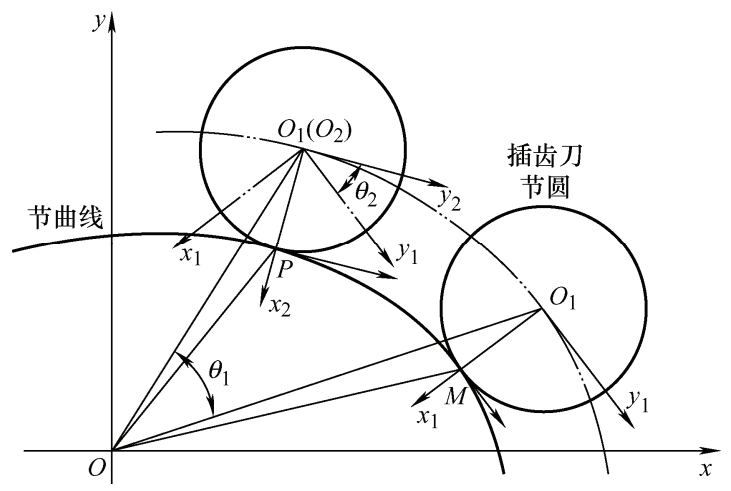

图 10 范成法原理 3

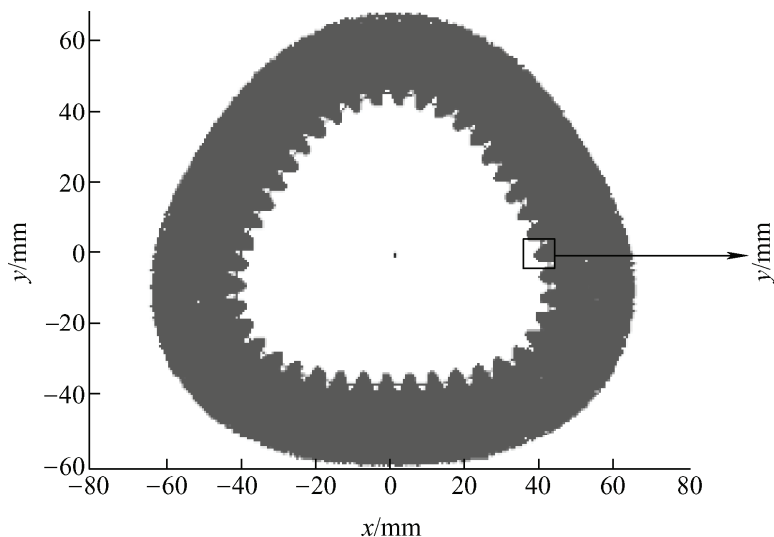

这两种方法在计算非圆齿轮齿廓时本质上并无 区别，本文以相对复杂的第二种范成方式为例简要 介绍基于范成法的非圆齿轮齿廓计算方法。

如图 11 所示, 设非圆齿轮所在坐标系为固定坐 标系 $O x y$; 坐标系 $O_{1} x_{1} y_{1}$ 与插齿刀固联; 坐标系 $\mathrm{O}_{2} x_{2} y_{2}$ 为插齿刀的随动坐标系, 且 $y_{2}$ 轴沿接触点切 线方向， $x_{2}$ 轴沿接触点法线方向；假设 $M$ 点为插刀 的初始点, 插齿刀由 $M$ 点沿节曲线纯滚动到 $P$ 点时, 转动角度为 $\theta_{2} ; \theta_{1}$ 为插齿刀由 $M$ 沿节曲线纯滚动到 $P$ 点时相对于固定坐标系 $O x y$ 的夹角。此时 $O_{2}$ 点坐 标为 $\left(x_{1}, y_{0}\right)$, 由以上关系, 设插齿刀具在固定坐标 系 $O x y$ 中的齿廓方程为 $r_{2}=\left[\begin{array}{lll}x_{2} & y_{1} & 1\end{array}\right]^{\mathrm{T}}$, 则插齿刀 具齿廓在随动坐标系 $\mathrm{O}_{2} x_{2} y_{2}$ 中运动的轨迹在坐标系 Oxy 中的包络方程表达为

$$
\begin{gathered}
\boldsymbol{r}=\boldsymbol{M}_{01} \boldsymbol{M}_{12} \boldsymbol{r}_{2} \\
\boldsymbol{M}_{12}=\left[\begin{array}{ccc}
\cos \left(\theta_{2}\right) & -\sin \left(\theta_{2}\right) & x_{0} \\
\sin \left(\theta_{2}\right) & \cos \left(\theta_{2}\right) & y_{0} \\
0 & 0 & 1
\end{array}\right] \\
\boldsymbol{M}_{01}=\left[\begin{array}{ccc}
\cos \left(\theta_{1}\right) & -\sin \left(\theta_{1}\right) & 0 \\
\sin \left(\theta_{1}\right) & \cos \left(\theta_{1}\right) & 0 \\
0 & 0 & 1
\end{array}\right]
\end{gathered}
$$

依式(18)运动插齿刀模型, 可得到如图 11 所示 的非圆齿轮包络结果。

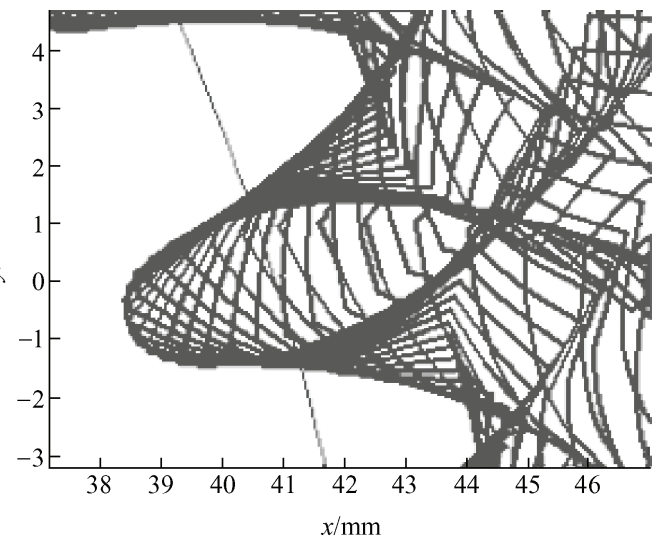

图 11 非圆齿轮包络结果图

但式(18)所表达的只是非圆齿轮齿廓的包络 线, 如图 11 中右侧放大图所示, 其包络的边界即为 非圆齿轮的齿廓。虽由包络图可以观察非圆齿轮齿 廓的大致形状, 但是齿廓特征点的坐标却无法依据 式(18)得出, 因此上述方法并不能直接计算出非圆 齿轮的齿廓特征点。为了能获取齿廓上的特征点, 现阶段研究主要有两种计算齿廓特征点的方法。

(1) 将齿廓特征点表示为产型轮轮廓间交点集 的子集, 如图 12 所示 ${ }^{[23]}$; 该方法通常先计算出相 邻产型轮轮廓间的交点集, 再通过一定的数据篮选 规则篎选出齿廓特征点集, 该方法所得的齿廓特征
点精度高，数量多，易于拟合，且拟合齿廓曲线与 实际齿廓形状最为接近。但该算法时间复杂度高, 在设计高精度的非圆齿轮齿廓曲线时，需要消耗大 量时间。

（2）为了降低算法的时间复杂度，部分研究简 化了非圆齿轮齿廓特征点提取算法, 将齿廓特征点 表示为产型轮轮廓与非圆齿轮节曲线多条等距线的 交点。如图 13 所示, 该方法首先在非圆齿轮的齿顶 曲线与齿根曲线之间绘制多条节曲线的等距线, 再 求出包络过程中各等距线与各产型轮轮廓间的交点 集, 最后篮选出齿廓特征点集。该算法经优化后比 
第一种算法所需的计算次数更少, 时间复杂度更低, 但在精度方面不如第一种算法 ${ }^{[25-26]}$ 。

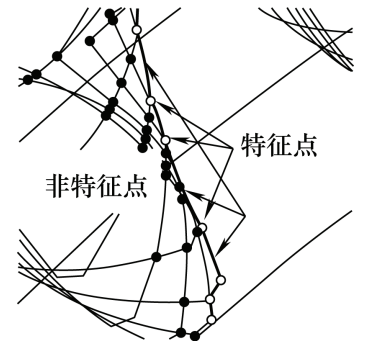

图 12 齿廓特征点提取 1

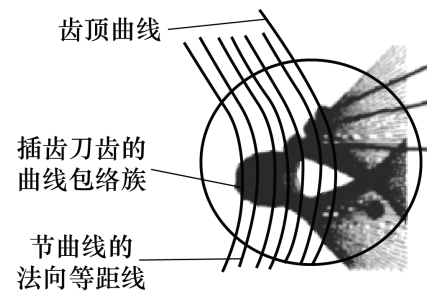

图 13 齿廓特征点提取 2
通过以上两种方法, 即可得到齿廓特征点集, 最后对点集进行样条拟合, 即可求得非圆齿轮齿廓 曲线。

\section{3 非圆齿轮的分析}

随着计算机仿真技术的迅猛发展, 通过仿真分 析软件判断非圆齿轮设计是否满足要求已逐渐成为 现阶段非圆齿轮设计过程中不可缺少的一环。通过 对非圆齿轮模型的仿真分析, 可在设计阶段验证设 计结果是否满足需求, 发现设计缺陷, 从而在设计 阶段提高成功率，节约了非圆齿轮研发的时间、经 济成本。现阶段, 非圆齿轮分析领域的研究主要可 分为运动学分析及动力学分析两部分。

(1) 非圆齿轮的运动学分析。

现阶段研究中, 非圆齿轮的运动学分析并不关心 非圆齿轮受力情况, 只研究非圆齿轮在指定工况下的 速度、加速度以及中心距变化是否满足需要。运动学 分析主要步骤如下: 首先建立非圆齿轮的三维模型, 再将模型导入 ADAMS、Pro/Mechanism、SolidWorks 等软件中, 建立非圆齿轮啮合联动模型, 模拟非圆齿 轮在实际工作环境中的运动, 并记录运动过程中角速 度、角加速度以及中心距的变化, 绘制曲线图, 最后 对曲线图进行分析, 得到非圆齿轮的运动学特性 ${ }^{[27-28]}$ 。

大量的研究基于此方法，对不同种类的非圆齿 轮进行了运动学分析。虽然不同形状的非圆齿轮, 其运动学分析结果不尽相同, 但多数研究中都得出 了非圆齿轮副的非匀变速传动特性会在传动中引起 不可避免的震动与冲击的结论 ${ }^{[27-28,67]}$ 。

(2) 非圆齿轮的动力学分析。

非圆齿轮的动力学分析理论上是通过求解微分 方程组得到非圆齿轮的受力与运动并加以分析的过 程。但由于动力学微分方程数量众多且含有大量非 线性项, 使得单靠求解微分方程组几乎无法得到非 圆齿轮动力学方程的解析解。因此, 现阶段主要是 依靠 ADAMS、ANASYS 等分析软件研究非圆齿轮 动力学特性的 ${ }^{[29]}$ 。动力学分析主要步骤可概括为:
首先建立非圆齿轮的三维几何模型, 并将非圆齿轮模 型导入有限元分析软件; 设置它们的位置、运动副、 驱动、负载等参数; 运行软件, 完成非圆齿轮的动力 学分析, 得到非圆齿轮的动力学仿真结果 ${ }^{[30-31]}$ 。

部分研究发现, 非圆齿轮在动力学分析过程中 从动轮转速的提高速度比主动轮扭矩的提高速度快 得多。通过分析后推测, 此现象可能是因为非圆齿 轮啮合运动时因为传动比变化导致的齿面分离、齿 面碰撞引起的 ${ }^{[32]}$ 。

\section{2 非圆齿轮的制造}

现阶段非圆齿轮的制造方法多种多样，可分为 靠模加工、线切割、数控插齿、数控滚齿、数控铣 齿等。靠模法每设计一套模具仅能加工一种非圆齿 轮, 制造成本高, 仅能满足单一非圆齿轮大批量生 产的需求，目前鲜有研究者关注。线切割技术虽能 加工几乎所有种类的非圆齿轮, 且加工效率高, 但 相比其他加工方法精度略低 ${ }^{[33-34]}$; 数控铣齿 ${ }^{[35]}$ 加工 精度较高, 但其加工效率低下。因此, 现阶段非圆 齿轮制造领域相关研究主要集中在加工精度、效率 均较高的数控插齿、滚齿技术上。

\section{1 数控插齿}

非圆齿轮数控插齿是运用日益成熟的计算机辅 助加工技术，从建立产型轮、毛肧模型开始仿真插 齿过程, 得到实际插齿过程中产型轮与毛肧的位置、 转角关系，从而自动生成数控插齿加工代码，最终 在数控插齿机中，完成非圆齿轮数控插齿加工。

现阶段研究中, 非圆齿轮的数控插齿加工可归 纳为如下步骤。

(1) 按需建立非圆齿轮毛肧模型(图 14)与产型 轮模型(图 15) ${ }^{[14,36]}$ 。

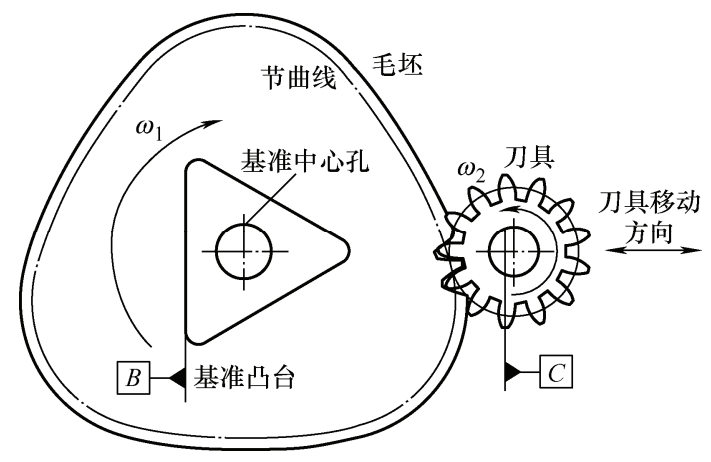

图 14 非圆齿轮的毛坏及基准部位

（2）依据范成法原理, 从多种加工策略中选择 最适合的加工策略, 如图 14、16 所示, 建立非圆齿 轮数控插齿加工联动模型 ${ }^{[37-38]}$ 。 
(3) 判断是否存在根切、顶切等 ${ }^{[39]}$, 逐步完善 加工策略, 生成数控加工代码, 以尽量减少非圆齿 轮试切加工造成的时间与经济损耗。

(4) 将毛胚与产型轮正确安装在数控插齿机 上, 具体位置如图 14 所示, 输入加工代码, 完成加工。

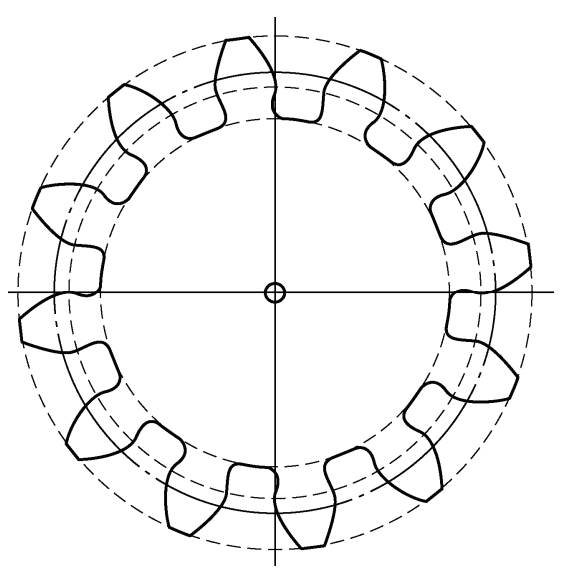

图 15 插齿刀模型

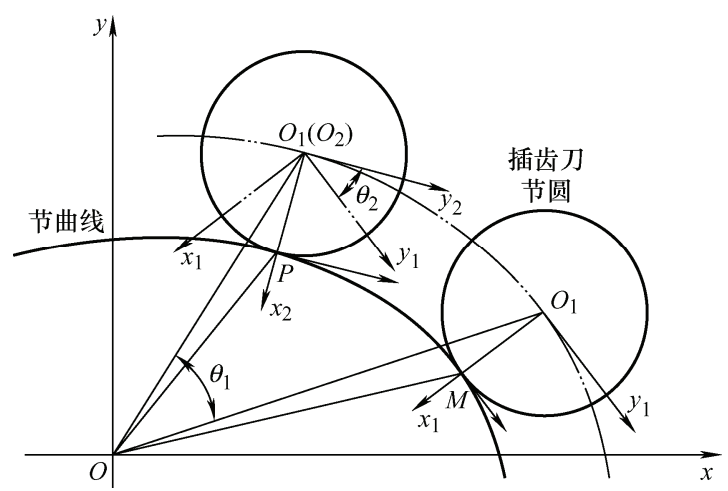

图 16 插齿刀与非圆齿轮纯滚动

现阶段大部分研究在插齿刀模型的选择上均选用 了标准插齿刀模型, 但也有部分研究依据实际需要, 使用了特定齿数、模数的插齿刀模型 ${ }^{[35]}$; 此外, 为了 能保证加工时毛胚与插齿刀间初始中心距、转角的准 确性, 较新研究中提出了在插齿刀刀柄及非圆齿轮毛 胚上设置基准面的方法, 在齿廓设计与非圆齿轮加工 时, 依据基准面对齐非圆齿轮与产型轮位置, 如图 14 所示。该方法解决了装夹过程中产型轮及非圆齿轮毛 胚难以确定相互位置, 导致加工误差增大的问题 ${ }^{[40]}$ 。

现阶段研究在插齿加工设备与策略选择上也做了 不少工作。有研究总结了现有 4 种不同的非圆齿轮插 齿加工策略, 其中包括: 插齿刀等转角、毛胚等弧长、 毛胚等转角以及毛肧等极角, 并通过仿真对比, 得出 了插齿加工时使用齿坏等弧长策略加工出的非圆齿轮 齿廓相比其他策略更加均匀, 精度更统一的结论 ${ }^{[37]}$ 。

现阶段研究指出, 加工过程中出现顶切根切主 要是因为传统三轴数控插齿机床的退刀方向在插齿 刀与非圆齿轮的联心线上, 而插齿过程中, 如图 17 所示, 插齿刀的节圆与工件节曲线的切点并不在联心
线上, 因而在非圆齿轮插齿加工时普遍存退刀干涉问 题, 且 $\beta$ 角越大, 干涉越明显 (大于三轴的数控插齿机 床能更好地调整退刀方向, 通过多轴控制, 完全消除 $\beta$ 角, 不存在此问题)。因此, 部分研究提出了改进退 刀策略, 以减小或消除退刀过程中导致退刀干涉的 $\beta$ 角 ${ }^{[41]}$ 。具体操作方法如图 18 所示, $O_{2}^{\prime}$ 为插齿刀加工 时的位置, $O_{2}^{\prime \prime}$ 为插齿刀运动到机床下止点并沿着非圆 齿轮轮坏与插刀中心连线方向移动一段距离后的位 置, 通过一段距离的让刀减小退刀过程中导致退刀干 涉的 $\beta$ 角, 实现了非圆齿轮的无干涉连续插齿加工 ${ }^{[42]}$ 。

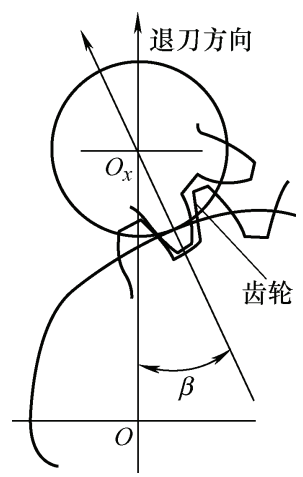

图 17 退刀干涉

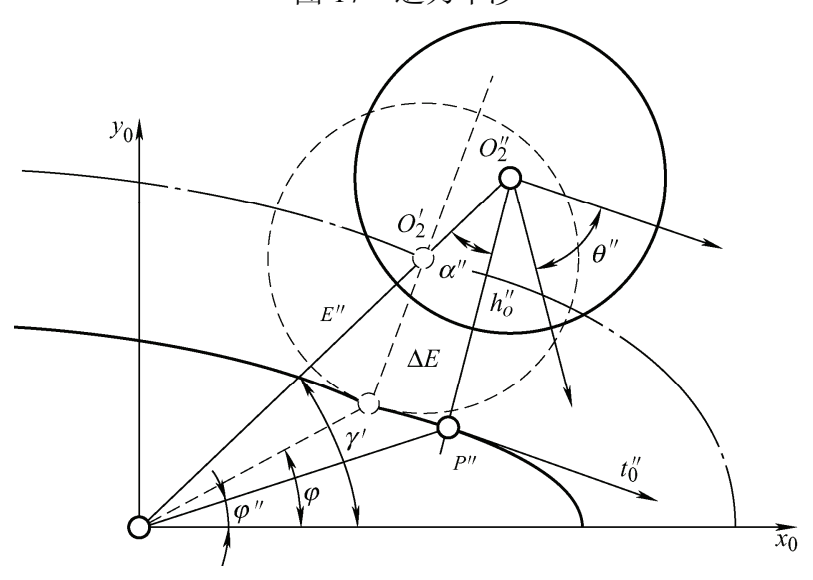

图 18 退刀时刀具和齿坏的位置关系

部分研究为了解决非圆齿轮插齿加工中存在齿 面加工余量分布不均的问题, 在粗切加工时, 如 图 19 所示, 使插刀粗切位置相对精切位置沿齿坏节 曲线法线向外偏移了一段距离, 保证径向进给切削 运动始终在节曲线法向上进行，实现了齿面加工余 量匀化 ${ }^{[43]}$ 。同时也消除了退刀过程中导致退刀干涉 的 $\beta$ 角, 实现了非圆齿轮无干涉连续插齿加工。

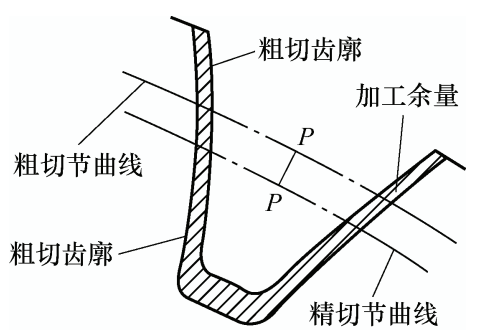

图 19 面加工余量匀化工艺原理 


\section{2 数控滚齿}

非圆齿轮数控滚齿技术与插齿技术部分相似, 但又有所不同。其相似之处在于: 滚齿技术同样是 基于计算机辅助加工技术, 需要建立滚齿刀与毛胚 模型, 通过基于范成原理的虚拟加工技术仿真滚齿 过程, 得到数控滚齿加工代码并导入数控滚齿机, 完成非圆齿轮的数控滚齿加工。但其不同之处在于 以下几点。

(1) 滚齿刀模型在滚齿联动模型中可等效为齿 条模型, 因此在构建产型轮模型时, 现阶段研究大 都是以齿条模型代替滚齿刀模型, 建立数控滚齿联 动模型的 ${ }^{[44-45]}$ 。

(2) 除开加工策略对加工精度的影响, 加工设 备, 特别是滚齿数控机床轴数, 对非圆齿轮滚齿加 工策略及精度影响非常大。因此应谨慎选择合理的 数控滚齿加工设备, 并依据加工设备, 选择适合的 加工策略。

部分研究对加工策略及滚齿加工数控机床的选 择进行了深入的探讨。它们总结了现有三种滚齿加 工策略：工件相对于滚刀等弧长运动、工件相对于 机床等转角运动以及滚刀相对于齿坏等转角运动, 并建立了 2 种数控滚齿机床联动模型—四轴联动 滚齿机床联动模型(图 20)及五轴联动滚齿机床联 动模型(图 21)。在尝试了不同的加工设备与加工 策略组合后, 通过仿真分析, 得出了在机床选择 方面, 五轴联动滚齿机床比四轴联动滚齿机床在 加工非圆齿轮方面精度更高, 震动更小, 更具优 势。而在众多加工策略选择中, 得到了每种策略 都有各自的优缺点与适用范围, 具体加工时可依据实 际情况与加工策略特性选择加工策略的结论 ${ }^{[46-47]}$ 。最 新研究中亦有研究者建立了六轴滚齿机床联动模 型 ${ }^{[48]}$, 然而并没有将其与 4 轴 5 轴数控滚齿机床 作对比。

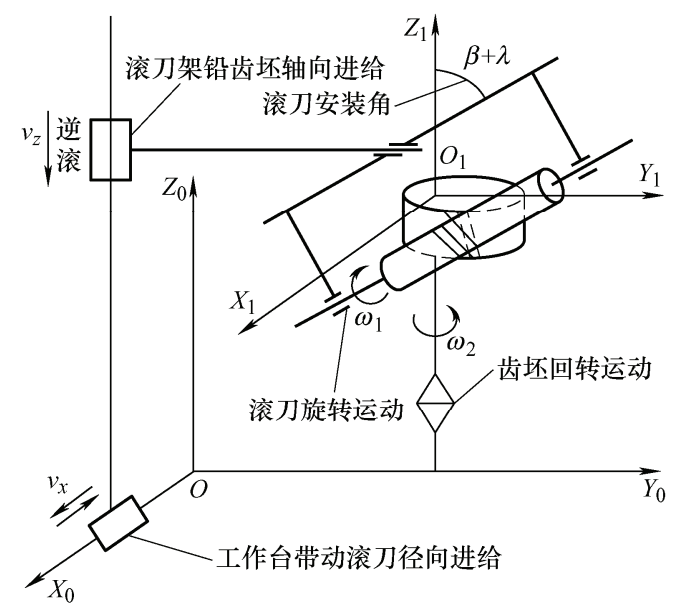

图 20 四轴滚齿加工联动示意图
此外, 部分研究为了解决部分四轴滚齿数控机 床刀具无轴向位移所导致的工作范围窄、负载不均 及滚刀齿间磨损等问题, 提出了如图 22 所示的两种 解决方法 ${ }^{[49]}$ : 第一种方法是在滚齿过程中保持产型 轮与毛胚的啮合点固定不变, 从而保证滚齿过程中 数控滚齿机床的稳定性, 提高滚齿精度; 第二种方 法是让产型轮与毛胚的啮合点在滚齿过程中沿滚齿 刀匀速运动, 让滚齿刀每个齿都参与到滚齿过程中, 从而延长滚齿刀整体寿命。

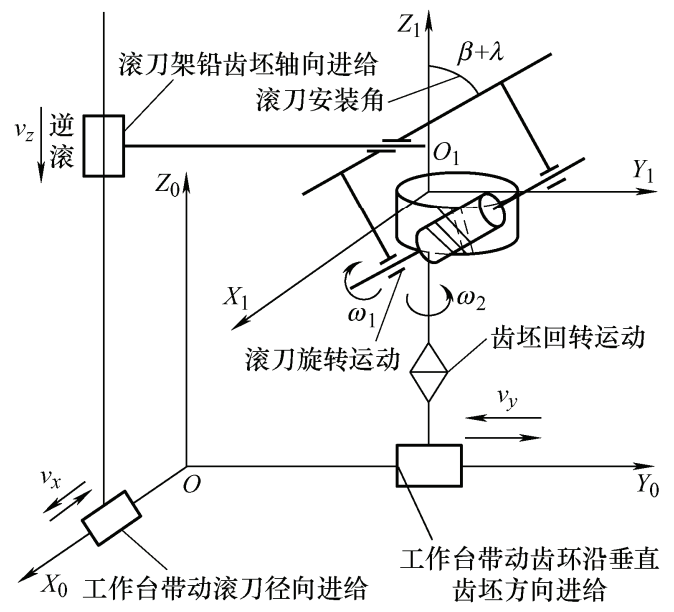

图 21 五轴联动滚齿加工联动示意图

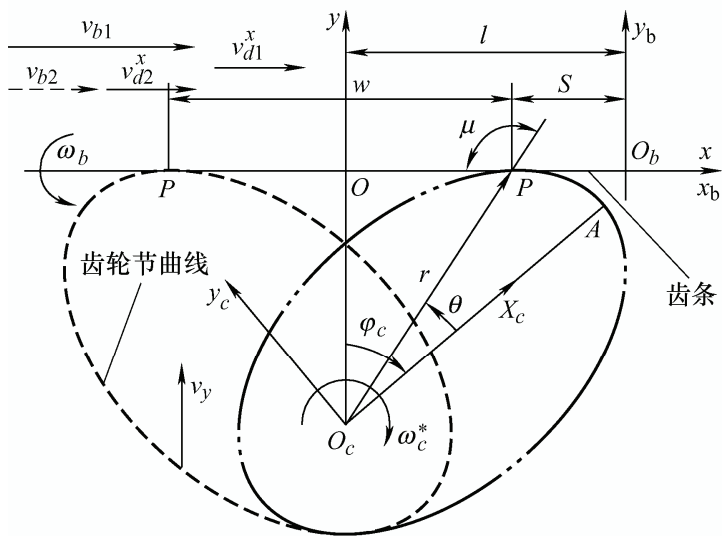

图 22 非圆齿轮四轴滚齿数控机床滚切优化示意图

\section{3 非圆齿轮的检测}

受制于非圆齿轮特殊性, 圆齿轮的精度标准、测 量方法与仪器一般不能直接套用在非圆齿轮上, 以至 于非圆齿轮误差检测技术发展缓慢, 目前尚处于初级 阶段。国内外关于非圆齿轮误差检测方法、检测仪器 和精度标准的研究非常有限, 远不如非圆齿轮的其他 领域研究热度高。在非圆齿轮检测领域, 按照检测误 差项性质，可分为单项误差检测和综合误差检测两大 类，其中单项误差中包含齿廓误差、齿距误差，而综 合误差中包含切向综合误差及径向综合误差。 


\section{1 单项几何误差检测}

\subsection{1 齿廓误差检测}

非圆齿轮的齿廓误差检测是运用的高精度探头 (图 23)或高分辨率摄像头等仪器采集如图 24 所示的 非圆齿轮实际齿廓特征点数据。之后可采用多种曲 线重建算法, 如在特征点邻域中取点并通过预估该 点法线的方法重建实际齿廓曲线 ${ }^{[50]}$, 或运用 NURBS 插值算法重建齿廓曲线 ${ }^{[51]}$ 等方法绘制实际齿廓曲 线, 通过研究中给出的曲线误差量化算法与理论齿 廓对比, 量化非圆齿轮齿廓误差, 从而确定被测非 圆齿轮误差大小。

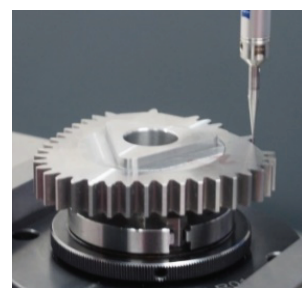

图 23 非圆齿轮齿廓的检测

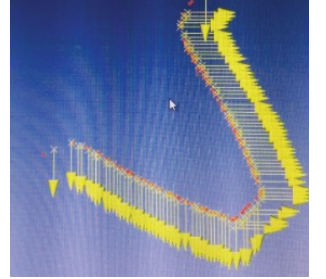

图 24 非圆齿轮的齿廓重建
部分工作进一步研究了非圆齿轮径向/切向齿 廓误差, 并绘出了解析图, 如图 25、26 所示。研 究将实际加工过程与非圆齿轮齿廓误差模型结合, 通过对解析图的分析, 总结出了非圆齿轮加工误差 特征码, 推导出了对单一误差或简单的两种误差组 合情况具备较准识别能力的加工误差成因图 ${ }^{[52-53]}$ 。

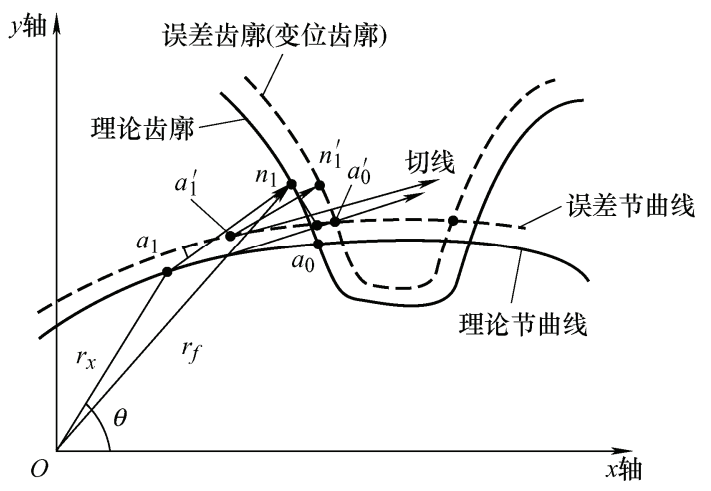

图 25 非圆齿轮径向齿廓误差解析图

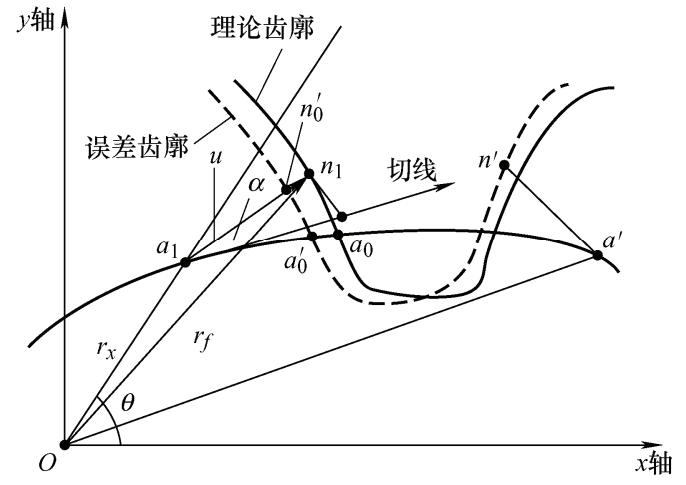

图 26 非圆齿轮切向齿廓误差解析图
依据成因图, 可在已知非圆齿轮齿廓几何误差数据 的情况下, 判断误差成因, 为加工方案的进一步优 化提供思路。

\subsection{2 齿距误差检测}

非圆齿轮的齿距误差检测是在圆齿轮齿距误差 检测基础上加以改进后的产物。由于非圆齿轮各齿 齿廓曲线不尽相同，为了使每个量棒与所测齿槽的 某侧齿廓的接触点都在节曲线上, 每种不同的非圆 齿轮进行跨棒距检测之前, 都需要设计一套该齿轮 专用的量棒 ${ }^{[54]}$ 。

如图 27 所示, $M\left(x_{0}, y_{0}\right)$ 点为第 $k$ 号逆时针齿 廓与节曲线函数的交点，同时也为量棒与齿廓在节 曲线上的交点, 且 $M$ 点处齿廓曲率为 $k_{0} ; N_{i}\left(x_{i}, y_{i}\right)$ 为第 $k$ 号顺时针齿廓上的点, $N_{i}$ 点处齿廓曲率为 $k_{i}$ 。 若量棒与第 $k$ 号顺时针齿廓交于点 $N_{i}$ 并在节曲线 上, 则量棒中心 $O_{i}(x, y)$ 到测量接触点 $M$ 和搜索点 $N_{i}$ 的距离可分别表示为

$$
\begin{aligned}
& L_{1 i}=\left|O_{i} M\right|=\sqrt{\left(x-x_{0}\right)^{2}+\left(y-y_{0}\right)^{2}} \\
& L_{2 i}=\left|O_{i} N\right|=\sqrt{\left(x-x_{i}\right)^{2}+\left(y-y_{i}\right)^{2}}
\end{aligned}
$$

其中, $L_{1 i}$ 和 $L_{2 i}$ 的长度随着顺时针齿廓上 $N_{i}\left(x_{i}, y_{i}\right)$ 点位置的变化而变化, 当: $L_{1 i}=L_{2 i}$ 时, $N_{i}$ 点和 $M$ 点即为量棒的与齿廓在节曲线上的两个交 点。此时, $r_{i}=L_{1 i}=L_{2 i}=\sqrt{\left(x-x_{0}\right)^{2}+\left(y-y_{0}\right)^{2}}$ 即为量 棒的半径, $O_{i}(x, y)$ 为量棒中心, 同理可得非圆齿轮 其他齿槽的量棒半径及中心坐标，从而制造出适合 待测非圆齿轮的量棒组。

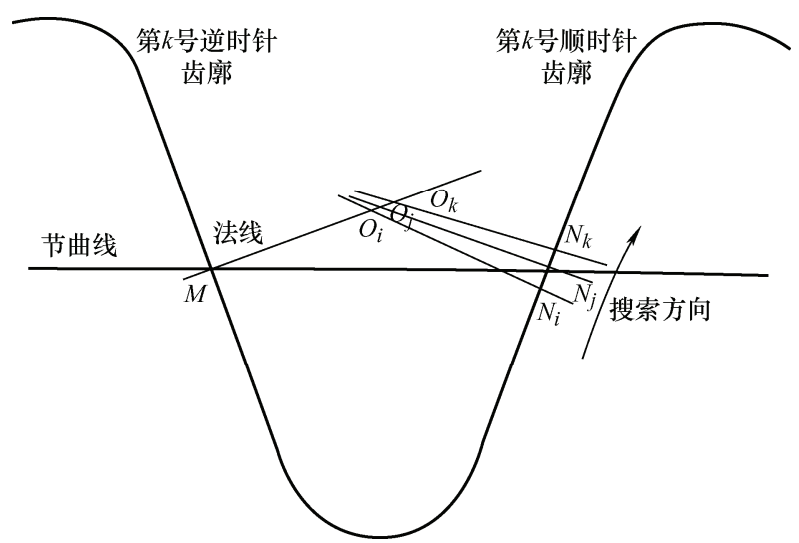

图 27 量棒测量接触点搜索原理图

得到适合该非圆齿轮的量棒组后，设第 $m$ 号齿 槽的量棒半径为 $r_{m}$, 中心点坐标为 $O_{m}\left(x_{m}, y_{m}\right)$; 第 $n$ 号齿槽应量棒半径为 $r_{n}$, 中心点坐标为 $O_{n}\left(x_{n}, y_{n}\right)$ 。 则可得第 $m$ 号齿槽与第 $n$ 号齿槽之间的跨棒距为

$$
L=\sqrt{\left(x_{m}-x_{n}\right)^{2}+\left(y_{m}-y_{n}\right)^{2}}+r_{m}+r_{n}
$$

$m \neq n$ 
其他齿槽的量棒尺寸与跨棒距均可用以上方法 算出, 即可上述方法可算出非圆齿轮的齿距误差。

还有研究设计出了如图 28 所示的某跨棒距检 测装置 ${ }^{[55]}$, 该装置通过自动化检测手段获取实际量 棒与非圆齿轮齿廓的几何位置, 计算理论跨棒距与 实际跨棒距间差值, 从而得到非圆齿轮齿距误差, 使得该方法不但避免了人工检测所带来的误差, 还 显著地提高了检测效率 ${ }^{[54]}$ 。

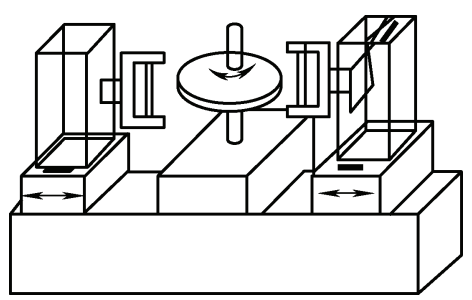

图 28 非圆齿轮的跨棒距测量装置

\section{2 综合误差检测}

\subsection{1 切向综合误差}

齿轮单面啮合测量法是检测齿轮切向综合误差 的一种重要方法 ${ }^{[1]}$ 。该方法通过测量非圆齿轮与标 准圆柱齿轮(可以用标准齿条、标准蜗杆代替)在理 论中心距下做有侧隙地单面啮合传动时实际转角与 理论转角间的差值, 从而计算出非圆齿轮的切向综 合误差。

如图 29 所示, 两个圆光栅同轴安装在非圆齿轮 与标准圆柱齿轮上, $\theta_{1} 、 \theta_{2}$ 为圆光栅测量的两个非 圆齿轮的实际转角; $A+\Delta A$ 为理论中心距, 其中 $\Delta A$ 为理论中心距变动量; 输入输出端之间的理论变传 动比为 $i$, 当标准圆柱齿轮的角位移为 $\theta_{1}$ 时, 非圆 齿轮的理论角位移可表示为 $\theta_{2}=\theta_{1} / i$, 通过对比实 际角位移, 此时非圆齿轮实际转角与理论转角的传 动误差为 $\sigma$, 即为非圆齿轮的切向综合误差 ${ }^{[17-18]}$ 。

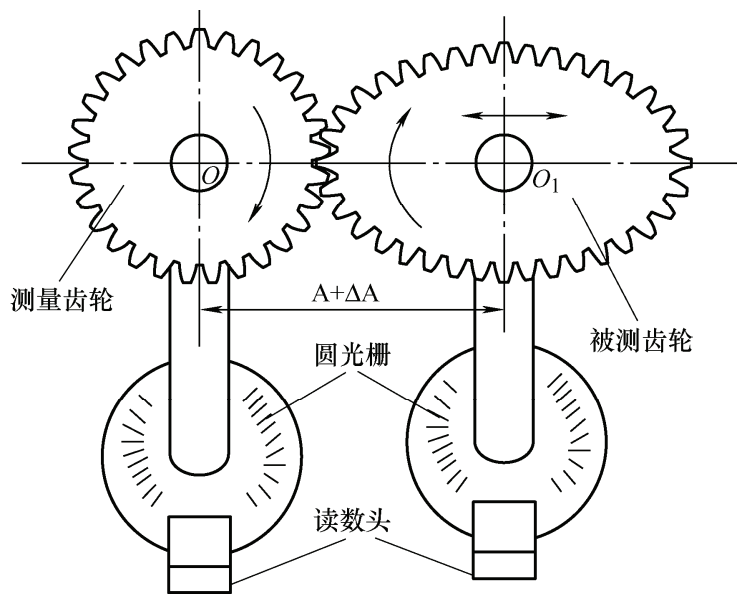

图 29 非圆齿轮单面啮合原理图

\section{2 .2 径向综合误差}

齿轮双面啮合测量法, 是一种检测齿轮径向综
合误差的重要方法。依据研究中检测方案设计的不 同可分为圆-非圆齿轮双面啮合检测与非圆-非圆双 面啮合检测，它们的区别在于被测非圆齿轮的啮合 对象是标准齿轮还是非圆齿轮。虽然以上两种方案 有区别，但它们的检测原理近乎相同。下面以圆非圆齿轮双面啮合检测为例，简要介绍非圆齿轮双 面啮合测量法。

圆-非圆齿轮双面啮合测量原理如图 30 所示, 被测非圆齿轮和标准圆齿轮做无侧隙的双面啮合运 动。如果两齿轮都是理想无偏差的, 那么两齿轮转 动时其中心距会严格按照非圆齿轮节曲线极径的变 化而变化。设被测非圆齿轮转角为 $\varphi$ 时其理论中心 距为 $A$, 即 $A$ 是 $\varphi$ 的函数。则理论中心距与被测非 圆齿轮的转角关系为: $A=A(\varphi)$ 。另一方面, 实际 中心矩 $A^{\prime}$ 可通过长光栅测得, 则 $\varphi$ 时中心距变动误 差为: $\Delta A=A^{\prime}-A(\varphi)$ 。此时 $\Delta A$ 就反映了被测非圆 齿轮在 $\varphi$ 时的径向综合误差。

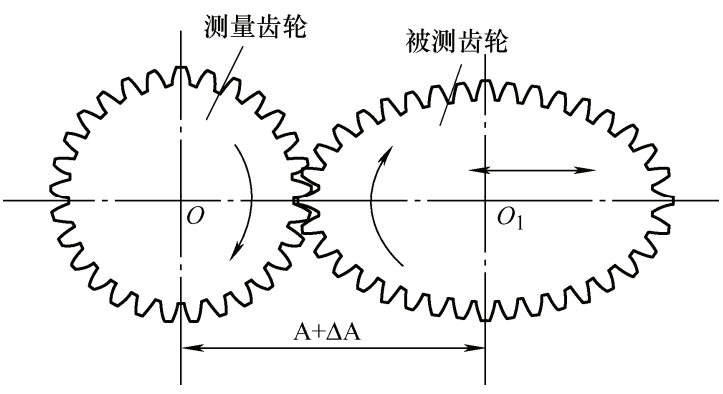

图 30 非圆齿轮双面啮合测量

在领域研究之初，并没有非圆齿轮专用的双面 啮合检测装置, 而是用圆齿轮的检测仪器加以改造 来测量非圆齿轮径向综合误差的。如通过机械式中 心距变动量测量仪(图 31)检测非圆齿轮副的中心距 变化量, 或通过机械式转角误差测量仪(图 32)检测 非圆齿轮副转角误差和空回，通过手动式节曲线形 状检查仪检测非圆齿轮节曲线误差。但此类检测仪 器都需人工操作，因此测量误差大、效率低。

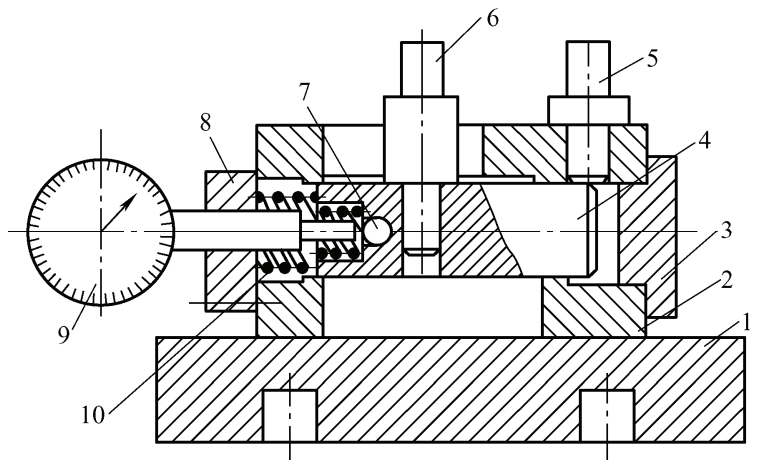

图 31 中心距变动测量仪

1. 底座 2. 壳体 3. 挡块 4. 轴 5. 非圆柱齿轮 1 6. 非圆柱齿轮 2 7. 钢球 8 . 挡块 9 . 表头 10 . 弹簧 


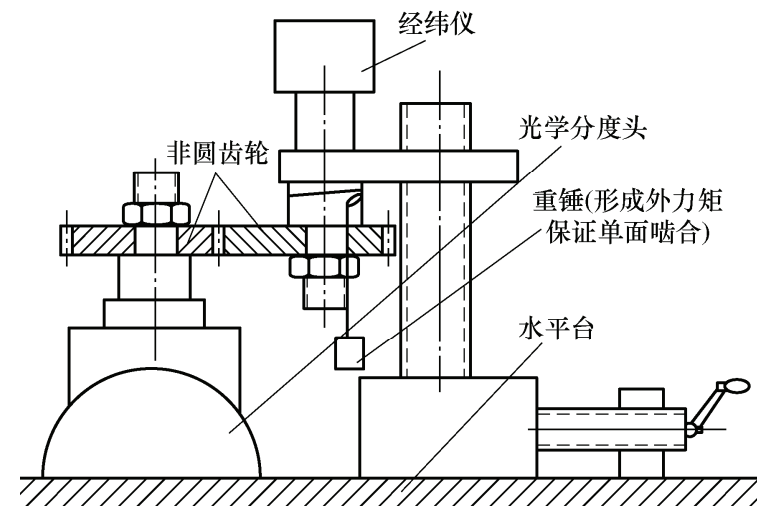

图 32 转角误差测量仪

随着自动化技术的发展, 现阶段研究中提出了 非圆齿轮综合误差自动化检测方法, 如图 33 所示, 通过将传统肉眼读数方式改为传感器采集数据, 再 传输到计算机, 最后由计算机进行数据处理、误差 计算和结果显示 ${ }^{[56]}$ 。

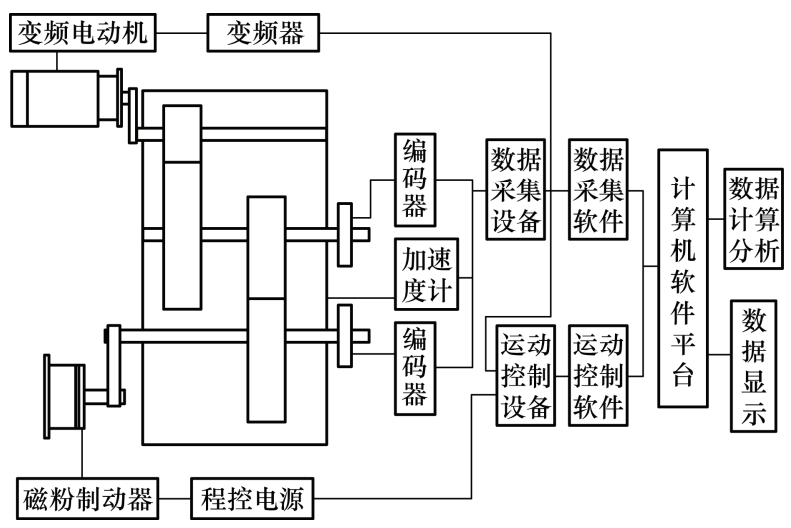

图 33 某非圆齿轮双面啮合检测仪总体方案图

目前较新研究中还设计了新型非圆齿轮双面啮 合装置, 该装置在啮合检测过程中能任意改变载荷 大小, 传动速度等, 从而实时监控主从动轮传动精 度、振动特性等参数 ${ }^{[57]}$, 使得检测出的非圆齿轮径 向误差更加贴近实际工况。

\section{4 非圆齿轮的应用}

非圆齿轮传动不仅具有齿轮传动精度高、效率 高、承载强、摩擦小、能高速传动等优点, 还能像 凸轮、连杆机构一样变中心距、变传动比传动, 实 现不同种类的函数输出。使得非圆齿轮不仅能够替 代传统凸轮、连杆机构, 甚至能通过与其他机构组 合, 实现更加复杂的运动, 具有广阔的应用前景与 研究价值。现阶段研究中非圆齿轮应用主要可概括 为三个方面。

(1) 替代凸轮、连杆或与之组合。

如图 34 所示, 非圆齿轮现阶段主要应用方向之
一就是替代传统凸轮、连杆机构, 或与凸轮、连杆 机构组合形成新的机构。相比传统凸轮、连杆机构, 改进后的机构具有传动精度高、效率高、承载强、 摩擦小、能高速传动等优点 ${ }^{[58-59]}$ 。

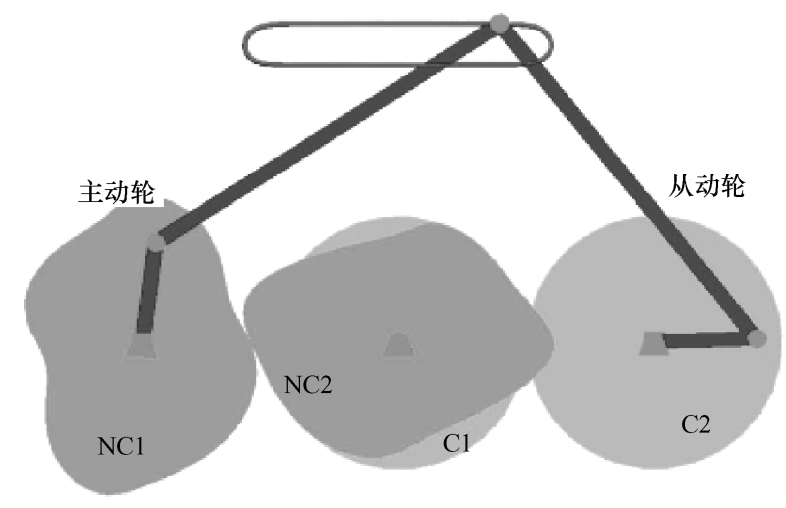

图 34 非圆齿轮-连杆机构

例如，在现阶段在农业机械领域中，非圆齿轮 除了被应用于简单的农用机械外，还被应用于自动 取苗分插、扎穴、施肥等具有复杂运动轨迹的大型 农业机械中一一使用基于非圆齿轮的旋转式机构取 代了传统往复式曲柄摇杆式机构, 使得改进后的机 构惯性更小, 结构更紧凑, 在高速作业下, 能保持 更高的运动精度。图 35 所示为一种基于非圆齿轮的 取苗分插机构。该机构由 5 个非圆齿轮、行星架及 两个栽植臂组成, 替代了由凸轮、连杆机构所组成 的传统分插机构，从而具有更合适的取苗轨迹及取 苗、推苗姿态 ${ }^{[60-62]}$ 。另有研究应用该机构原理, 设 计出了基于非圆齿轮的扎穴及施肥机构, 用非圆齿 轮替代了原同类机构中的凸轮、连杆, 同样具有良 好的效果 ${ }^{[63]}$ 。

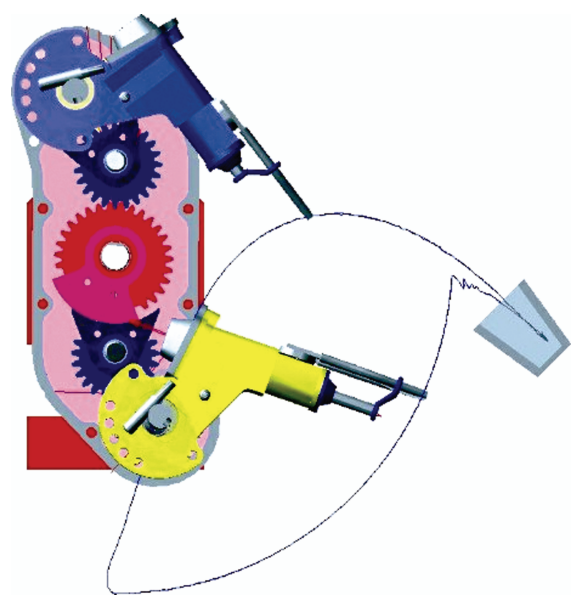

图 35 非圆齿轮匀速间歇齿轮传动机构

纺织机械领域中，也涌现出了一大批基于非圆 齿轮的新型纺织机构, 如基于椭圆齿轮的曲柄摇杆 引纬机构 ${ }^{[64]}$ 、基于偏心齿轮和共轭双叶非圆齿轮的 导线机构 ${ }^{[65]}$, 以及非圆齿轮连杆组合传动的新型针 
杆与挑线驱动机构 ${ }^{[66]}$ 等, 它们大量替代了传统纺织 机械中的凸轮、连杆机构, 提高了纺织机械精度与 效率，延长了机构使用寿命。

亦有研究将非圆齿轮与曲柄滑块机构结合, 构 成了如图 36 所示的基于非圆齿轮的曲柄滑块式驱 动机构 ${ }^{[67]}$, 该机构能实现滑块的非线性运动输出。 可用于血液葲驱动装置中, 在心脏手术时为身体提 供脉动血流 ${ }^{[68]}$ 。

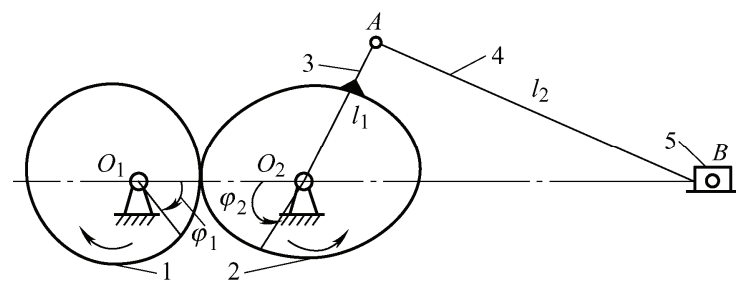

图 36 非圆齿轮-曲柄滑块式机构

1. 主动轮 2. 从动轮 3 . 曲柄 4. 连杆 5 . 滑块

(2) 应用非圆齿轮几何形状特殊性。

非圆齿轮的另一个主要应用方向, 即发挥其外轮 廓非圆特色，设计出应用于不同领域的非圆齿轮机构。

例如, 在液压泵领域, 现阶段研究充分运用了 非圆齿轮的几何特性, 设计出了如图 37 所示的非圆 叶片差速泵 ${ }^{[69]}$ 、非圆行星轮䈋 ${ }^{[70]}$ 等液压泵机构, 有 效地改善了传统液压洜机构容积效率低, 流量波动 大, 压力波动大, 噪声大等问题。部分研究还在液 压泵中加入了如图 38 所示的非圆控制机构, 充分利 用了非圆齿轮外轮廓特性, 使得该控制机构在输入 端角速度与方向恒定情况下自动切换输出端的正反 转, 实现了抽油泵机构自动换向功能 ${ }^{[71]}$ 。

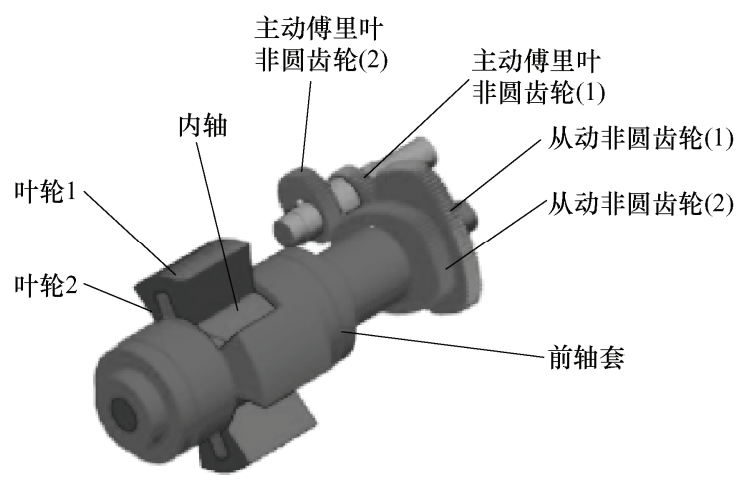

图 37 非圆齿轮差速泵

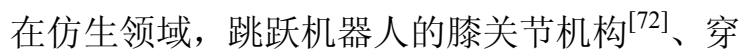
戴式膝关节辅助步行康复仪 ${ }^{[73]}$ 以及六足仿生机器 人的腿部传动系统 ${ }^{[74-75]}$ 等机构中也都应有了非圆齿 轮的几何特性。现阶段非圆齿轮与仿生学的高度融 合, 使得人们能设计出与人类膝关节、蜘蛛关节等 更加近似的非圆齿轮机械关节机构, 进一步提高了 现有机械关节的灵活度、传动性能及承载能力。

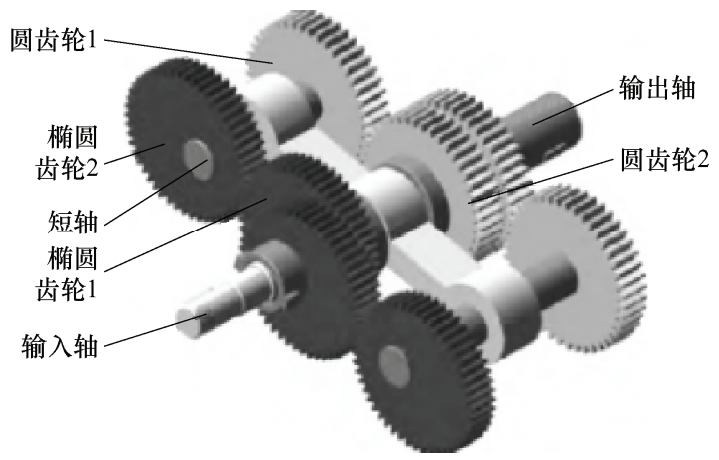

图 38 非圆齿轮换向装置

非圆齿轮几何形状的不对称性同样广泛应用于 各类惯性补偿机构中。例如用于驱动内燃机辅助飞 轮, 以平衡内燃机中飞轮加速和减速时产生的扭矩 波动 ${ }^{[76]}$; 或在各类机械关节机构中充当重力平衡补 偿机构 ${ }^{[77]}$, 解决了目前弹簧-质量重力平衡机构在平 衡旋转摆领域的不足。

(3) 应用非圆齿轮变速比、变中心距传动特性。

非圆齿轮所变传动比、变中心距传动的特性使 其能输出各种周期函数、间歇运动 ${ }^{[78-79]}$, 不仅能让 各类变速机构、间歇机构的变速过程更平滑，亦能 让震动机构震动得更规律, 使得非圆齿轮特别适用 于各类差变速、震动领域。

在差速领域, 研究者们相继设计出了如图 39、 40 所示的非圆差速器, 它们较传统差速器具有更大 的锁紧系数, 更强的防滑功能, 从而提高了车辆的 越野通过性 ${ }^{[80]}$ 。在变速器领域, 由于非圆齿轮变速 传动时具传动比变化平滑, 因此有研究设计出了基 于非圆齿轮的无级变速器, 该变速器能有效缓解变 速时机构内部产生的瞬时冲击, 相较传统变速器具 有更加平滑的变速过程 ${ }^{[81]}$ 。

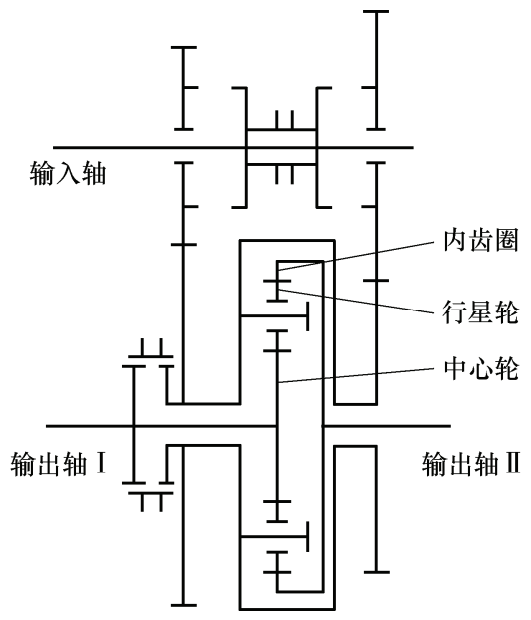

图 39 带有轴间差速器的非圆齿轮分动器

在间歇运动领域, 部分研究将非圆齿轮用于非正 弦振动驱动结晶器中, 通过非圆齿轮在传动时中心距 的变化产生特定的振动，在锻造大型钢板时能减少拉 
漏, 减轻振痕, 提高铸坯表面质量和拉坏速度 ${ }^{[82]}$ 。

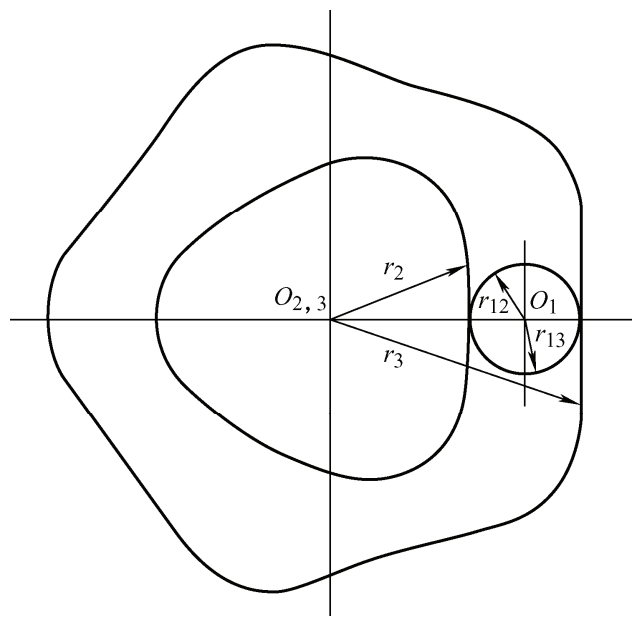

图 40 非圆行星齿轮差速器

\section{5 结论与展望}

通过归纳现阶段非圆齿轮研究, 可看出非圆直 齿轮在设计与分析、制造、检测及应用领域均有相 当多学者开展了大量有意义、有启发性的研究。本 节在充分总结现有研究成果与不足的基础上, 对非 圆齿轮的 4 大领域的总结与展望如下。

(1) 非圆齿轮设计与分析领域。

主要包括节曲线设计、齿廓设计以及分析三部 分。其中节曲线设计主要有两种方法: 曲线变性拼 接拟合法理论易于理解、易操作, 但面对复杂的节 曲线时, 往往需要花费大量时间拟合出所需曲线。 微分几何推算法是非圆齿轮节曲线的一般化设计方 法, 但在求解变中心距非圆齿轮节曲线时, 通过该 方法建立的高阶偏微分方程难以求解, 使得这类方 法也有一定局限性。在非圆齿轮齿廓设计领域, 范 成法作为应用最为广泛的非圆齿轮齿廓求解方法, 具有诸多优点, 但该方法中齿廓特征点集提取精度 与计算时间成正比, 现阶段齿廓特征点提取算法往 往需要较长时间才能提取出高精度的齿廓特征点 集。非圆齿轮的分析也是非圆齿轮设计不可缺少的 一部分, 通过对非圆齿轮的运动学与动力学分析, 能在设计之初就对非圆齿轮运动学与动力学性能有 所了解, 避免设计出不满足需求的非圆齿轮, 提高 了设计效率, 降低了设计成本, 但是现阶段非圆齿 轮的分析仅能判断非圆齿轮是否符合要求, 却鲜有 研究能依据分析结果提出修改建议。

非圆齿轮设计领域未来在如下方向均有可研究 之处。

(1) 考虑到不同基曲线具有各异的几何特性, 如
何利用不同基曲线，更好更快地设计出满足需求的 节曲线。

(2) 突破非圆齿轮设计领域现状, 引入其他数学 工具, 应用旋量理论 ${ }^{[32]}$ 或尝试其他的数学工具对非 圆齿轮设计领域进行研究。

(3) 优化非圆齿轮齿廓提取算法, 降低齿廓特征 点提取算法的时间复杂度。

(4) 如何使非圆齿轮的运动学与动力学分析结 果反馈到非圆齿轮节曲线与齿廓设计中, 为非圆齿 轮设计提出优化意见。

(2) 非圆齿轮制造领域。

主要研究以插齿与滚齿为主。其中数控滚齿加 工效率高, 精度高, 但是其对加工设备要求也高(需 要 4 6 轴数控滚齿机床), 且只能加工一定曲率范 围内的非圆外齿轮。数控插齿具有设备成本低( $3 、 4$ 轴数控机床就可加工), 精度高, 适用性强等优点, 能加工非圆内齿轮、内凹齿轮, 但其加工效率不如 滚齿效率高。此外, 非圆齿轮的滚齿与插齿均有大 量不同的加工策略, 致使众多研究尝试了不同的加 工设备与加工策略组合, 并分析了它们的优劣。可 以看出, 非圆齿轮的制造领域本质上是对设备与加 工策略的选择的研究, 然而现阶段, 对于某一非圆 齿轮, 并没有一套详细的设备与加工策略选择策略, 且现阶段加工策略的选择并没有考虑加工过程中的 切削速度、切削力以及温度变化等因素。

非圆齿轮制造领域未来在如下方向均有可研究 之处。

(1) 研究非圆齿轮几何特性与加工设备、策略间 的关系, 为不同非圆齿轮提供最适合的加工设备与 策略。

(2) 将更多因素, 如切削速度、切削力以及温度 变化等加入研究中, 使得加工设备、策略的选择更 加复合实际情况。

(3) 非圆齿轮检测领域。

主要研究非圆齿轮齿廓误差、齿距误差、切向综 合误差与径向综合误差, 现阶段研究主要以改进圆齿 轮检测方法、工具为主, 缺乏非圆齿轮专用的检测理 论、方法与工具, 更缺乏统一的精度标准。在现阶段 研究中, 齿廓误差检测虽然测量精度高, 但是耗时较 长、受限于探头的大小, 在检测时部分齿轮因其模数 较小需破坏齿轮的完整性才能完成检测。非圆齿轮的 齿距检测中, 针对每个不同的齿轮都要设计不同直径 的量棒组, 通用性较差、不经济。综合误差检测中也 存在仪器精度不高、细节不完善等问题。

非圆齿轮检测领域未来在如下方向均有可研究 之处。 
(1) 制定一套非圆齿轮精度标准。

(2) 完善检测工具、发展新的检测方法, 如高精 度的非破坏性齿廓检测仪器、通用性强的非圆齿廓 跨棒距检测量棒与方法、高自动化、多功能的非圆 齿轮径/切向综合误差检测仪等。

(4) 非圆齿轮的应用。

主要有三个方向：替代原有凸轮、连杆机构的 应用, 发挥非圆齿轮几何特殊性的应用以及对非圆 齿轮传动时变传动比变中心距特性的应用。

虽然非圆齿轮与凸轮、连杆机构相比具有诸多 优势, 但是受到研发人员的思维定式, 以及设计成 本、加工成本、精度等诸多条件制约, 尚不能广泛 应用于各类机械中。所以, 非圆齿轮应用的发展除 了需要研究者勇于创新, 打破思维定势, 想方设法 将非圆齿轮用于各类场景中以外, 还需要研究者在 非圆齿轮的设计与分析、加工、检测等方面持之以 恒地努力, 以期有效降低非圆齿轮应用门槛, 从而 推动非圆齿轮研究更好的发展与更加广泛的应用。

\section{参 考 文 献}

[1] 陈定方, 孔建益, 杨家军, 等. 现代机械设计师手册(上 册)[M]. 北京: 机械工业出版社, 2014.

CHEN Dingfang, KONG Jianyi, YANG Jiajun, et al. Handbook of modern mechanical designers (Volume 1) [M]. Beijing: China Machine Press, 2014.

[2] ADDOMINE M, FIGLIOLINI G, PENNESTRI E. A landmark in the history of non-circular gears design: The mechanical masterpiece of dondi's astrarium[J]. Mechanism \& Machine Theory, 2018, 122: 219-232.

[3] CHENG Kai, NIU Zhichao, WANG R C, et al. Smart cutting tools and smart machining: Development approaches, and their implementation and application perspectives[J]. Chinese Journal of Mechanical Engineering, 2017(30): 1162-1176.

[4] 叶军, 陈建能, 赵雄, 等. 一种新型类偏心圆非圆齿轮设 计及其应用[J]. 中国机械工程, 2018, 29(5): 565-571， 578. YE Jun, CHEN Jianneng, ZHAO Xiong, et al. Design and applications of generalized eccentric noncircular gears[J]. China Mechanical Engineering, 2018, 29(5): 565-571, 578.

[5] XIE Z H, QIU D M. Study on high-order elliptical gear parametric design, automatic modeling and motion simulation[J]. Applied Mechanics and Materials, 2012, 271-272: 1147-1154.

[6] 张瑞, 吴序堂, 聂钢, 等. 高阶变性椭圆齿轮的研究与 设计 $[\mathrm{J}]$. 西安交通大学学报, 2005(7): 726-730.
ZHANG Rui, WU Xutang, NIE Gang, et al. Study and design on high-order deformed elliptic gears[J]. Journal of Xi'an Jiaotong University, 2005(7): 726-730.

[7] LIN Chao, XIA Xiguang, LI Peiliu. Geometric design and kinematics analysis of coplanar double internal meshing non-circular planetary gear train[J]. Advances in Mechanical Engineering, 2018, 10(12): 1-12.

[8] 刘永平, 王鹏, 县喜龙, 等. Pascal 蜗线型齿轮齿廓设 计 $[\mathrm{J}]$. 机械传动, 2015，39(3): 50-52.

LIU Yongping, WANG Peng, XIAN Xilong, et al. Design of the tooth profile of pascal curve gear[J]. Mechanical Transmission, 2015, 39(3): 50-52.

[9] 刘生林, 黄先祥, 吴序堂. 用三次样条进行非圆齿轮节曲 线设计的研究[J]. 机械设计, 1999(5): 21-22, 40, 43.

LIU Shenglin, HUANG Xianxiang, WU Xutang. Study on the pitch curve design of non-circular gears with cubic spline[J]. Mechanical Design, 1999(5): 21-22, 40, 43.

[10] FANGHELLA P. Kinematic synthesis and design of non-circular gears through a symbolic-numeric modeling approach[C]//ASME International Design Engineering Technical Conferences \& Computers \& Information in Engineering Conference. September 24-28, 2005, Long Beach, California. New York: DETC, 2005: 781-789.

[11] FIGLIOLINI G, STACHEL H, ANGELES J. Base curves of involute cylindrical gears via aronhold's first theorem $[\mathrm{J}]$. Proceedings of the Institution of Mechanical Engineers Part C-Journal of Mechanical Engineering Science, 2016, 230(7-8): 1233-1242.

[12] 俞高红, 虞佳萍, 童俊华, 等.一种共轭凹凸型非圆齿 轮机构的设计 [J]. 中国机械工程, 2016, 27(16): 2155-2159, 2165.

YU Gaohong, YU Jiaping, TONG Junhua, et al. Design of a conjugate concave-convex non-circular gear mechanism[J]. China Mechanical Engineering, 2016, 27(16): 2155-2159, 2165.

[13] 刘琴琴, 吕刚, 邓星桥. 基于样条插值方法的非圆齿轮 节曲线设计缺陷的修正 $[\mathrm{J}]$. 机械传动，2018，42(5): 90-95.

LIU Qinqin, LÜ Gang, DENG Xingqiao. Modification of the pitch curve design defect of non-circular gear based on spline interpolation method[J]. Journal of Mechanical Transmission, 2018, 42 (5): 90-95.

[14] ZHENG Fangyan, HUA Ling, HAN Xinghui. The mathematical model and mechanical properties of variable center distance gears based on screw theory[J]. Mechanism 
and Machine Theory, 2016, 101: 116-139.

[15] SUN Ke, ZHENG Fangyan, CHEN Dingfang, et al. Design method and analysis of non-circular gear based on variable ratio function[J]. Journal of Mechanical Transmission, 2013, 8351: 544-552.

[16] DAI Jiansheng. Screw algebra and kinematic approaches for mechanisms and robotics[M]. London: Springer, 2014..

[17] LITVIN F L. Gear geometry and applied theory[M]. USA: Prentice-Hall, 1994.

[18] 吴序堂. 齿轮啮合原理[M]. 西安: 西安交通大学出版 社, 2009.

WU Xutang. Theory of gearing[M]. Xi'an: Xi'an Jiaotong University Press, 2009.

[19] 高雪强, 葛敬侠. SolidWorks 中的非圆齿轮实体建模方 法研究[J]. 工程图学学报, 2009, 30(4): 189-192.

GAO Xueqiang, GE Jingxia. Study on parametric solid modeling of non-circular gear in solidworks[J]. Journal of Engineering Graphics, 2009, 30(4): 189-192.

[20] LOZZI A. Non-circular gears-graphic generation of involutes and base outlines[J]. Proceedings of the Institution of Mechanical Engineers Part C-Journal of Mechanical Engineering Science, 2000, 214(3): 411-422.

[21] 刘永平, 王鹏, 李佳. 解析法设计椭圆齿轮齿廓[J]. 机 械设计与制造，2015，288(2)：214-216， 220.

LIU Yongping, WANG Peng, LI Jia. Design the elliptical gear tooth profile via analytical method[J]. Machinery Design \& Manufacture, 2015, 288(2): 214-216, 220.

[22] 胡赤兵, 姚洪辉. 基于 MATLAB 及 Pro/E 的参数化非 圆齿轮设计 $[\mathrm{J}]$. 机械传动, 2010，34(7): 33-35，38.

HU Chibing, YAO Honghui. The parametric design research of noncirle gear based on matlab and pro/e[J]. Journal of Mechanical Transmission, 2010, 34(7): 33-35, 38.

[23] 武传宇, 金玉珍, 贺磊盈. 基于包络特征的非圆齿轮齿 廓计算方法研究[J]. 中国机械工程, 2008, 255(15): 1796-1799.

WU Chuanyu, JIN Yuzhen, HE Leiying. Numerical algorithm of tooth profile of noncircular gear based on the characteristics of cutter envelope[J]. China Mechanical Engineering, 2008, 255(15): 1796-1799.

[24] LYASHKOV A A, PANCHUK K L, KHASANOVA I A. Automated geometric and computer-aided non-circular gear formation modeling $[\mathrm{J}]$. Journal of Physics: Conference Series, 2018, 1050: 012049.

[25] 李建刚, 吴序堂, 李泽湘. 基于插齿数值计算模型的非 圆齿轮根切分析[J]. 农业机械学报, 2007(6): 138-142.
LI Jiangang, WU Xutang, LI Zexiang. Analysis of tooth undercutting in noncircular gears based on numerical computation model of shaping[J]. Journal of Agricultural Machinery, 2007(6): 138-142.

[26] 李建刚, 吴序堂, 毛世民, 等. 非圆齿轮齿廓数值计算 的研究 [J]. 西安交通大学学报, 2005(1): 75-78.

LI Jiangang, WU Xutang, MAO Shimin, et al. Numerical computation of tooth profile of noncircular gear[J]. Journal of Xi' an Jiaotong University, 2005(1): 75-78.

[27] 张健, 饶鹏, 郑涁, 等. 非圆齿轮设计及其运动学分析 [J]. 机械研究与应用，2018，31(2)：38-42.

ZHANG jian, RAO Peng, ZHENG Bin, et al. Design and kinematics analysis of non-circular gear[J]. Mechanical Research \& Application, 2018, 31(2): 38-42.

[28] 刘永平, 董长斌, 安建民, 等. 新型抽油机换向装置结 构设计及运动仿真分析 $[\mathrm{J}]$. 机械传动, 2017, 41(9): 115-118, 134.

LIU Yongping, DONG Changbin, AN Jianmin, et al. Analysis of motion simulation and structure design of the reversing device of new pumping unit[J]. Mechanical Transmission, 2017, 41(9): 115-118, 134.

[29] HU Chibing, TIAN Fangyong, JIANG Yancang. Elliptical gears parametric design and motion simulation[J]. Advanced Materials Research, 2010, 139-141: 1206-1210.

[30] CHEN Jianneng. Establishment \& verification of dynamics mode of seedling pushingin transplanting mechanism with planetary elliptic gears[J]. Transactions of the CSAE, 2003, 19(5): 71-76.

[31] SUN Chuanqiong, SUN Guoxing, REN Aihua, et al. The kinematic analysis of heart-shaped gear continuously variable transmission[J]. Applied Mechanics and Materials, 2010, 37-38: 1489-1492.

[32] LIU X, NAGAMURA K, IKEJO K. Analysis of the dynamic characteristics of elliptical gears[J]. Journal of Advanced Mechanical Design Systems and Manufacturing, 2012, 6(4): 484-497.

[33] ZHANG Min, KONG Deyong, Zhao Jiali, et al. Solid modeling methods and wire-cutting process simulation of non-circular gears[J]. Applied Mechanics and Materials, 2011, 86: 730-734.

[34] HU Chibing, WANG Yazhou, KONG Deyong, et al. The solid modeling and simulation for non-circular gear based on pro/e[J]. Advanced Materials Research, 2011, 230-232: 506-511.

[35] ZHENG Fangyan, HAN Xinghui, LIN Hua. Design and 
manufacture of new type of non-circular cylindrical gear generated by face-milling method[J]. Mechanism and Machine Theory, 2018, 122: 326-346.

[36] 侯东海, 刘忠明, 吴序堂. 用工具斜齿条法加工斜齿非 圆齿轮的啮合理论模型 $[\mathrm{J}]$. 机械工程学报, 2003(8): 49-54.

HOU Donghai, LIU Zhongming, WU Xutang. Meshing theory analysis model for the manufacturing of helical non-circular gear by the helical tooling rack generating method [J]. Journal of Mechanical Engineering, 2003(8): 49-54.

[37] LIU Youyu. Study of optimal strategy and linkage-model for external non-circular helical gears shaping[J]. Proceedings of the Institution of Mechanical Engineers, Part C: Journal of Mechanical Engineering Science, 2015, 229(3): 493-504.

[38] LI Bo, HE Jun, ZHENG Fangzheng, et al. Research on three-axis linkage shaping technology for non-circular internal gears[J]. China Mechanical Engineering, 2016(6): 1455-1460, 1483.

[39] 何贵平, 胡赤兵, 靳岗, 等. 外啮合非圆齿轮加工模型 及根切特性研究 [J]. 兰州理工大学学报, 2006(6): 40-43.

HE Guiping, HU Chibing, JIN Lan, et al. Investigation of mathematical model for machining of externally meshed noncircular gears and characteristics of their undercut[J]. Journal of Lanzhou University of Technology, 2006(6): 40-43.

[40] ZHENG Fangyan, HUA Lin, HAN Xinghui. Linkage model and manufacturing process of shaping non-circular gears[J]. Mechanism and Machine Theory, 2016, 96, 192-212.

[41] 熊镇芹, 吴序堂, 毛世民, 等. 非圆齿轮插齿加工中的 退刀干涉机理及规避方法研究[J]. 机械工程学报, 2002(3): 152-155.

XIONG Zhenqin, WU Xutang, MAO Shimin, et al. Research on the mechanism and the avoidance method of cutting interference in non-circular gear shaping[J]. Chinese Journal of Mechanical Engineering, 2002(3): 152-155.

[42] ZHENG Fangyan, HUA Lin, HAN Xinghui, et al. Synthesis of indexing mechanisms with non-circular gears[J]. Mechanism and Machine Theory, 2016, 105: 108-128.

[43] 韩江, 高婷, 李大柱, 等. 非圆齿轮齿面加工余量匀化 工艺研究与实现 $[J]$. 中国机械工程, 2018, 29(9):
1031-1038

HAN Jiang, GAO Ting, LI Dazhu, et al. Sesearch and implementation of tooth surface machining allowance uniform distribution processing technology of non-circular gears[J]. China Mechanical Engineering, 2018，29(9): 1031-1038

[44] 谭伟明, 胡赤兵, 洗伟杰, 等. 非圆齿轮滚切最简数学 模型及其图形仿真[J]. 机械工程学报, 2001(5): 26-29. TAN Weiming, HU Chibing, XIAN Weijie, et al. Concise mathematical model for hobbing non-circular gear and its graphic simulation[J]. Chinese Journal of Mechanical Engineering, 2001(5): 26-29.

[45] TIAN Fangyong, HU Chibing, JIANG Yancang. The simplest mathematical model and simultaneous-control structure for hobbing helical non-circular gear[J]. Applied Mechanics and Materials, 2010，42: 284-288.

[46] 史勇, 王生泽. 斜齿非圆齿轮滚齿加工过程仿真研究 [J]. 机械设计，2015，32(4): 19-25.

SHI Yong, WANG Shengze. Simulation research on hobbing machining of helical non-circular gear[J]. Journal of Mechanical Design, 2015，32(4): 19-25.

[47] 胡赤兵, 丁和艳, 严克明, 等. 非圆斜齿轮滚切加工 $\mathrm{CNC}$ 联动控制方案 $[\mathrm{J}]$. 中国机械工程, 2004(24): 15-18. HU Chibing, DING Heyan, YAN Keming, et al. Simultaneous-control methods of $\mathrm{CNC}$ for hobbing noncircular helical gears[J]. China Mechanical Engineering, 2004(24): 15-18.

[48] LIU Youyu, DIAO Jiesheng. Six-axis linkage strategy and its models for non-circular helical gears based on diagonal hobbing[J]. Strojniski Vestnik-Journal of Mechanical Engineering, 2015, 61(5): 330-340.

[49] XIA Lian, LIU Youyu, LI Dazhu. A linkage model and applications of hobbing non-circular helical gears with axial shift of hob $[\mathrm{J}]$. Mechanism and Machine Theory, 2013, 70: 32-44.

[50] 高雯雯. 基于全齿测量的非圆齿轮误差分析 $[\mathrm{D}]$. 哈尔 滨：哈尔滨工业大学, 2016.

GAO Wenwen. Research on the error of non-circular gears based on the all teeth measurement method[D]. Harbin: Harbin University of Technology, 2016.

[51] 韩江, 高婷, 江本赤, 等. 非圆齿轮齿廓特性分析及偏 差测量方法研究 $[J]$. 电子测量与仪器学报, 2016, 30(10): 1520-1533.

HAN Jiang, GAO Ting, JIANG Benchi, et al. Tooth profile characteristics analysis and deviation measurement 
study of non-circular gear[J]. Journal of Electronic Measurement and Instrument, 2016, 30(10): 1520-1533.

[52] DAI Jianqing, WANG Shengze. Research on single point error quantization algorithm of non-circular gear tooth profile[J]. Machine Design \& Research, 2017, 33(4): 68-71.

[53] DAI Jianqing, WANG Shengze. Error detection and classification of tangential radial single machining of non-circular gear[J]. EDP Sciences, 2017, 41(2): 88-94.

[54] 刘永平, 孙旋. 非圆齿轮跨棒距测量的数学模型研究 [J]. 机械设计与制造，2014(11): 29-32.

LIU Yongping, SUN Xuan. Study on the mathematical model for measuring the span of non-circular gears[J]. Machinery Design \& Manufacture, 2014(11): 29-32.

[55] LIU Yongping, SUN Xuan. Mathematical model research of across the great distance measurement for non-circular gear[J]. Machinery Design \& Manufacture, 2014， 5(1): 43-56.

[56] LI Bo, ZHANG Hongxiang, CHEN Dingfang, et al. Measurement of composite error of pitch curve of non-circular gear based on $\mathrm{C}++$ and matlab[J]. Human Centered Computing, 2016, 9567: 215-225.

[57] 刘永平, 县喜龙, 王鹏. 非圆齿轮动态特性测试装置与 实验分析 $[\mathrm{J}]$. 机械传动, 2015，39(9): 126-128.

LIU Yongping, XIAN Xilong, WANG Peng. Dynamic characteristic testing device and experiment analysis of non-circular gear[J]. Mechanical Transmission, 2015, 39(9): 126-128.

[58] MUNDO D, GATTI G, DOONER D B. Optimized five-bar linkages with non-circular gears for exact path generation[J]. Mechanism and Machine Theory, 2009, 44(4): 751-760.

[59] BARI J M, CRANE C D, DOONER D B, et al. The design of a single degree of freedom open-loop spatial mechanism that incorporates geared connections[J]. Design and Manufacturing, 2009(4): 631-638.

[60] 俞高红, 陈志威, 赵匀, 等. 椭圆-不完全非圆齿轮行 星系蔬菜钵苗取苗机构的研究[J]. 机械工程学报, 2012, 48(13): 32-39.

YU Gaohong, CHEN Zhiwei, ZHAO Yun, et al. Study on vegetable plug seedling pick-up mechanism of planetary gear train with ellipse gears and incomplete non-circular gear[J]. Journal of Mechanical Engineering, 2012, 48(13): 32-39.

[61] 李革, 应孔月, 张继钊, 等. 基于秧针静轨迹的分插机
构非圆齿轮求解 $[\mathrm{J}]$. 机械工程学报, 2016, 52(1): 64-71. LI Ge, YING Kongyue, ZHANG Jizhao, et al. Computation method of non-circular gear based on seedling needle tip point's static trajectory in transplanting mechanism[J]. Journal of Mechanical Engineering, 2016, 52(1): 64-71.

[62] YE Bingliang, YI Weiming, YU Gaohong, et al. Optimization design and test of rice plug seedling transplanting mechanism of planetary gear train with incomplete eccentric circular gear and non-circular gears[J]. International Journal of Agricultural \& Biological Engineering, 2017, 10(6): 43-55.

[63] 王金武, 刘亚华, 王金峰，等. 全椭圆齿轮行星系液态 肥深施机构优化设计与试验[J]. 农业机械学报, 2012, 43(10): 59, 60-65.

WANG Jinwu, LIU Yahua, WANG Jinfeng, et al. Optimization design and experiment of liquid-fertilizer applying deep-fertilization mechanism for planetary elliptic gears[J]. Journal of Agricultural Machinery, 2012, 43(10): $59,60-65$.

[64] 陈建能, 赵雄, 徐宾, 等. 椭圆齿轮一曲柄摇杆引纬机 构的运动学建模与特性分析[J]. 中国机械工程, 2007, 235(19): 2294-2297, 2302.

CHEN Jianneng, ZHAO Xiong, XU Bin, et al. Establishment of kinematics models and performance analysis of elliptic-gear \& crank-rocker weft insertion mechanism[J]. China Mechanical Engineering, 2007, 235(19): 2294-2297, 2302.

[65] WANG Ying, CHEN Jianneng, TAO Honglian, et al. Modeling and analysis of traverse mechanism with eccentric gear and conjugated two-lobed non-circular gear[J]. Textile Research Journal, 2016, 87(17) : 2106-2116.

[66] 沈俊. 非圆齿轮针杆驱动机构运动学反求设计 [D]. 杭 州: 浙江理工大学, 2017.

SHEN Jun. Reserve kinematics design of needle bar driving mechanism with non-circulae gear[D]. Hangzhou: Zhejiang University of Technology, 2017.

[67] 贺敬良, 吴序堂, 李建刚. 变性椭圆齿轮连杆机构的运 动特性及齿轮副设计 [J]. 机械工程学报, 2004(1): 62-65.

HE Jingliang, WU Xutang, LI Jiangang. Kinematic characteristics and gear pair design for deformd elliptical geared linkage mechanism[J]. Chinese Journal of Mechanical Engineering, 2004(1): 62-65. 
[68] OtTAVIANO E, MUNDO D, DANIELI G A, et al. Numerical and experimental analysis of non-circular gears and cam-follower systems as function generators[J]. Mechanism and Machine Theory, 2008, 43(8): 996-1008.

[69] 陈明, 王广林, 刘福利, 等. 叶片差速泵偏心圆-非圆 齿轮驱动系统的研究 [J]. 机械工程学报, 2005(3): 98-101.

CHEN Ming, WANG Guanglin, LIU Welfare, et al. Study of eccentric circular- noncircular gears driing system of differential velocity vanes pump[J]. Chinese Journal of Mechanical Engineering, 2005(3): 98-101.

[70] DING Hong. Application of non-circular planetary gear mechanism in the gear pump[J]. Advanced Materials Research, 2012, 591-593: 2139-2142.

[71] 刘永平, 陈向宇, 孙旋, 等. 抽油机用非圆行星轮系换向 装置的设计与仿真 $[\mathrm{J}]$. 机械设计与制造，2018(3)：60-63. LIU Yongping, CHEN Xiangyu, SUN Xuan, et al. Design and simulation of non-circular planetary gear train reversing device for pumping unit[J]. Machinery Design \& Manufacture, 2018(3): 60-63.

[72] OKADA M, TAKEDA Y. Synthesis and evaluation of non-circular gear that realizes optimal gear ratio for jumping $\operatorname{robot}[\mathrm{C}] / /$ Intelligent Robots and Systems (IROS), November 3-11, 2013, Tokyo. Tokyo: IEEE/RSJ International Conference on IROS， 2013： 5524-5529.

[73] ZHU Yong, NAKAMURA M, ITO N, et al. Study of wearable knee assistive instruments for walk rehabilitation[J]. Journal of Advanced Mechanical Design Systems and Manufacturing, 2012, 6(2): 260-273.

[74] KE Junghuang, SHEN Chiangchen, MENG Ching, et al. A bio-inspired hexapod robot with noncircular gear transmission system $[\mathrm{C}] / /$ Advanced Intelligent Mechatronics (AIM), July 11-14, 2012, Kachsiung, Taiwan, China. Washington: IEEE, 2012: 33-38.

[75] LIU Zheng, ZHUANG Hongchao, GAO Haibo, et al. Static force analysis of foot of electrically driven heavyduty six-legged robot under tripod gait[J]. Chinese Journal of Mechanical Engineering, 2018，31: 63.

[76] DOONER D B, ZAMBRANAM N, GMBH V D B. Use of non-circular gears for crankshaft torque balancing in ic engines[J]. In International Conference on Gears, 2004, 1904: 197-212.

[77] BIJLSMA B G, RADAELli G, HERDER J L. Design of a compact gravity equilibrator with an unlimited range of motion[J]. Journal of Mechanisms and Robotics, 2017, 9(6): 061003.

[78] LITVIN F L, GONZALEZ-PEREZ I, FUENTES A, et al. Tandem design of mechanisms for function generation and output speed variation[J]. Computer Methods in Applied Mechanics and Engineering, 2009, 198(5-8): 860-876.

[79] LITVIN F L, GONZALEZ-PEREZ I, FUENTES A, et al. Design and investigation of gear drives with non-circular gears applied for speed variation and generation of functions[J]. Computer Methods in Applied Mechanics and Engineering, 2008， 197(45-48): 3783-3802.

[80] 贾巨民, 高波. 越野汽车分动器非圆行星差速器概念模 型[J]. 中国机械工程，2008，19(24): 3003-3005.

JIA Jumin, GAO Bo. A conceptive noncircular planetary differential for off- road vehicles[J]. China Mechanical Engineering, 2008, 19(24): 3003-3005.

[81] HEBBALE K, LI D, ZHOU J, et al. Study of a non-circular gear infinitely variable transmission[C]// ASME 2014 Dynamic Systems and Control Conference, October 22-24, 2014, San Antonio, TX, USA. New York: ASME, 2014: DSCC2014-6083.

[82] 张兴中, 李宪奎, 郑学然, 等. 椭圆齿轮驱动结晶器非 正弦振动的研究[J]. 机械工程学报, 2004(11): 178-182. ZHANG Xingzhong, LI Xiankui, ZHENG Xueran, et al. Study of non-sinusoidal oscillation of mold driven by ellipse gears[J]. Chinese Journal of Mechanical Engineering, 2004(11): 178-182.

作者简介: 李渤涛, 男, 1988 年出生, 博士研究生。主要研究方向为智 能设计与制造、机械工程、非圆齿轮、人工智能、计算机科学与技术。 E-mail: botao.li@foxmail.com

陈定方(通信作者), 男, 1946 年出生, 教授, 博士研究生导师。主要研 究方向为计算机辅助设计、人工智能与专家系统、科学计算可视化与计 算机仿真、基于网络的虚拟设计及制造、智能结构与智能材料。

E-mail: dfchen@mail.whut.edu.cn 